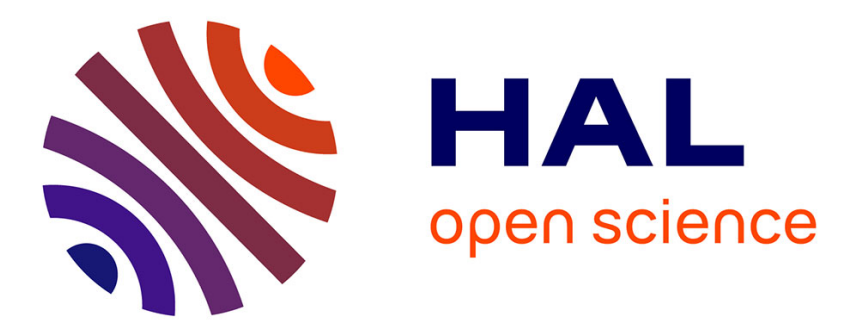

\title{
Energy management in Wireless Sensor Networks: A survey
}

\author{
Junaid Ahmed Khan, Hassaan Khaliq Qureshi, Adnan Iqbal
}

\section{To cite this version:}

Junaid Ahmed Khan, Hassaan Khaliq Qureshi, Adnan Iqbal. Energy management in Wireless Sensor Networks: A survey. Computers and Electrical Engineering, 2014, Volume 41, January 2015, pp. 159-176. 10.1016/j.compeleceng.2014.06.009 . hal-01283728

\section{HAL Id: hal-01283728 \\ https://hal.science/hal-01283728}

Submitted on 17 Mar 2016

HAL is a multi-disciplinary open access archive for the deposit and dissemination of scientific research documents, whether they are published or not. The documents may come from teaching and research institutions in France or abroad, or from public or private research centers.
L'archive ouverte pluridisciplinaire HAL, est destinée au dépôt et à la diffusion de documents scientifiques de niveau recherche, publiés ou non, émanant des établissements d'enseignement et de recherche français ou étrangers, des laboratoires publics ou privés. 


\title{
Energy Management in Wireless Sensor Networks: A Survey
}

\author{
Junaid Ahmed Khan $^{\mathrm{a}}$, Hassaan Khaliq Qureshi ${ }^{\mathrm{a}}$, Adnan Iqbal ${ }^{\mathrm{b}}$ \\ ${ }^{a}$ School of Electrical Engineering and Computer Science \\ National University Of Science and Technology, H-12, Islamabad, Pakistan \\ E-mail:10mseejunaidk@seecs.edu.pk,hassaan.khaliq@seecs.edu.pk \\ ${ }^{b}$ Namal College Mianwali, Pakistan \\ E-mail: adnan.iqbal@namal.edu.pk
}

\begin{abstract}
Energy management in Wireless Sensor Networks (WSNs) is of paramount importance for the remotely deployed energy stringent sensor nodes. These nodes are typically powered by attached batteries. Several battery-driven energy conservation schemes are proposed to ensure energy efficient network operation. The constraints associated to the limited battery capacity shifted the research trend towards finding alternate sources by harvesting ambient energy. This survey presents a high level taxonomy of energy management in WSNs. We analyze different battery-driven energy consumption based schemes and energy harvesting based energy provision schemes. We also highlight the recent breakthrough of wireless energy transference to a sensor node as an alternative to typical batteries. We recommend to take into account recent energy provisioning advancements in parallel with the traditional energy conservation approaches for a sensor network while designing energy efficient schemes.
\end{abstract}

Keywords: Wireless Sensor Networks, Energy Management, Energy Conservation, Energy Harvesting Sensors, Wireless Energy Transfer

\section{Introduction}

Energy management in WSNs is defined as the set of rules to manage various energy supply mechanisms and then efficient consumption of the provided energy in a sensor node. The overall aim should be to manage energy in such a way that no node becomes energy deficient and the network is operational perpetually. It is important for a sensor node to have an efficient energy management scheme for the limited source as well as the application requirement should be managed in accordance to the available energy source. Energy is considered as a scarce resource for a sensor node, specifically when a node is deployed in a remote region and once it depletes the available energy, it is almost impossible to provide supplant energy [1]. Therefore, a balanced energy management between the supply and the load is required in order to avoid energy deficiency in a network.

The objective of this survey is to provide awareness to the research community regarding the various energy management schemes. In order to get a full insight of the discussion, we classify energy management in WSNs on the basis of two main aspects. First, we shall discuss issues related with various energy supply mechanisms for a sensor node and then we shall discuss different approaches and the set of protocol designs based on energy consumption.

The most common power source for a sensor node is battery $[2,3]$, which can either be replaced or recharged based on the situation where it is deployed [4]. The limited battery supply constraints led many researchers to develop alternate energy provisioning mechanisms by utilizing ambient energy [5-9]. By harvesting ambient energy, a sensor network can have near perpetual operation. However, there exist limitations to energy harvesting based approaches as there might exist scenarios where a node suffers from limited harvesting opportunity than its power requirements [1]. In such scenarios, a node can become energy deficient, 


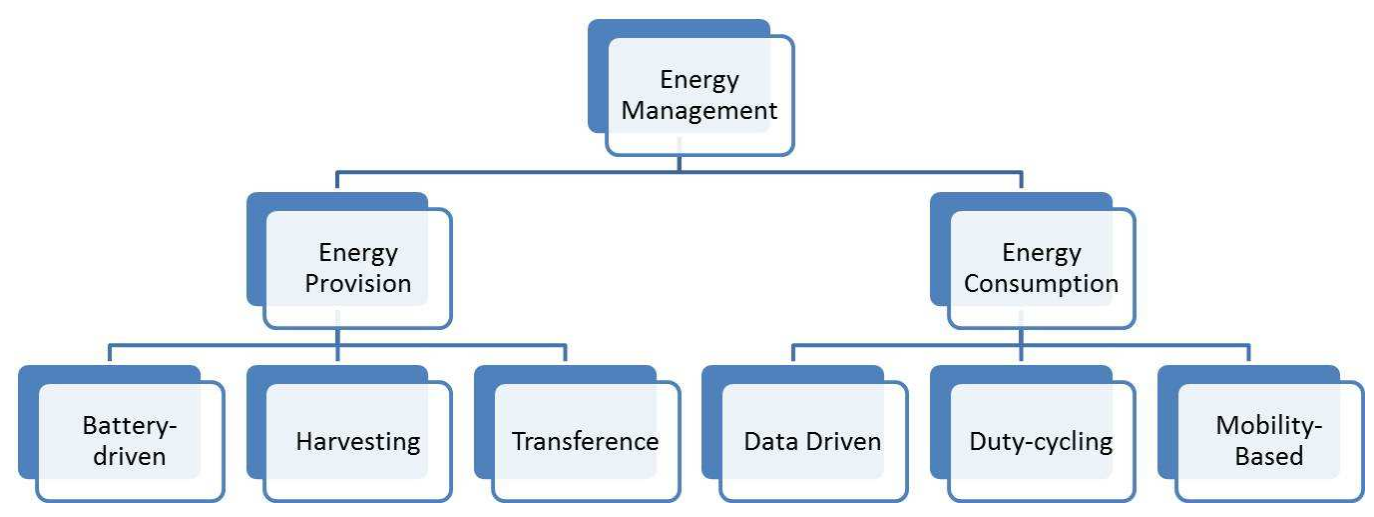

Figure 1: High level taxonomy

thus affecting the overall network performance.

Recently, energy transference based approaches are proposed, in which energy can be transferred on the field to an energy deficient node [10-15]. These energy deficient nodes are deployed in locations with limited harvesting opportunities from the environment. The wireless energy transfer is now considered as the most promising technology. However, besides energy supply mechanisms, there is also a need to study various approaches and protocols regarding efficient energy consumption in a node. Most of energy management efforts in literature mainly focus towards efficient node's energy consumption [16-28]. The aim is to use algorithms, which takes into account the limited energy supply constraints as well as develop applications with minimum energy consumption. However, the combination of efforts related to both energy supply and energy consumption opens path towards more promising and efficient energy management approaches in WSNs.

In this survey, we define a high level taxonomy of energy management approaches and in order to have a better insight of how to make energy management more efficient. Our findings reveal that the proper course is to consider energy provision as well as efficient energy consumption before proposing any energy management scheme. The efforts currently made in this regard can further steer the research trend towards more improved directions. For this purpose, this survey can be very useful in developing more effective energy management schemes. Moreover, it also provides a baseline toward improved extension of the existing energy management schemes.

This paper is organized as follows. In the next section, we proposed a high level taxonomy of the energy management schemes. In the third section, we will continue the discussion further into various energy provisioning mechanisms based on battery supply with their respective examples. In the fourth section, energy harvesting based provisioning techniques are discussed followed by the recent approaches on energy transference in the fifth section. In the sixth section, we will have a discussion on the various energy consumption based energy management approaches by stating relevant examples. In the end, we shall conclude the paper by providing insight into open research problems.

\section{High level taxonomy}

In this section, we describe a high level taxonomy of how to take energy management into consideration while designing or developing an algorithm. Energy management is based on two design considerations; energy provision and energy consumption as shown in Figure 1. We shall have a brief discussion on the generalized energy management schemes by breaking the taxonomy. The energy provision based schemes can be further classified into energy sourced by batteries, energy harvested from the environment and energy 


\begin{tabular}{ccc}
$\begin{array}{c}\text { Battery-Driven } \\
\text { Smartdust }\end{array}$ & $\begin{array}{c}\text { Transference } \\
\text { David D. L. }\end{array}$ \\
\hline 1998 & 2003 & 2008 \\
\hline & Harvesting & \\
& Rahimi M.
\end{tabular}

Figure 2: Evolution of energy provision technologies for sensor networks from battery-driven smartdust project [44] to Rahimi M. [7] study of energy harvesting and then David D. L's energy transference experiment in 2008 [10]

transference based schemes. The battery powered nodes are further classified on the basis of either fixed / replaceable battery supply or rechargeable battery. Similarly, the harvested energy also varies from different sources, of which, typical examples are solar, wind energy, thermal power, etc [29, 30]. The recent advancement in the field of WSNs evolved with the breakthrough of energy transference based supply [13]. The sources such as magnetic resonance, reflected solar energy, microwaves/ Radio Frequency (RF) Energy, and (Light Amplification by Stimulated Emission of Radiation) LASER power serves as the basics for energy transference based schemes [15, 31-33]. The sole purpose of each technology is to scavenge for alternate energy sources to provide as much surplus energy as possible to improve network lifetime.

On the other hand, lots of efforts have been made to develop efficient energy management schemes on the basis of energy consumption. These schemes can be typically divided into duty cycling based, data-driven and mobility based schemes. In duty cycling based schemes, nodes alternates between sleep and wake-up modes in order to achieve efficient energy utilization [17-25, 25-27, 34-36]. In data-driven approaches, several schemes are adopted to reduce data or to predict data, keeping accuracy at a certain level $[28,37,38]$. On the other hand, mobility based schemes use a mobile sink or a mobile relay depending on its behavior, which can be used as part of the environment or part of the network. Several algorithms are proposed for efficient energy utilization in each case [39-43]. However, here we provide a brief summary of energy management schemes with the help of the taxonomy tree shown in Figure 1. We will have a detailed discussion on each sub classification of this taxonomy in the subsequent sections. Figure 2 highlights a more generic chronological order of the advancements regarding energy provisioning technology starting from battery driven sources initiated by the smartdust project [44]. The authors in [7] studied the feasibility of energy harvesting from ambient environment. In 2008, David D. L. [10] initially demonstrated the breakthrough of wireless transfer of energy to a sensor node deployed in the field.

\section{Energy Provisioning Approaches based on Battery Supply}

In almost majority of the available sensor nodes, such as Mica, Telos and Iris [45], the primary power supply is fixed battery. Battery supply is considered as the most viable solution for the sensor nodes deployed in remote regions. It is mainly due to the low power requirement of nodes and its compatibility with the compact sized typical AA size battery. Figure 3 shows an example of battery driven crossbow's TelosB sensor node [46]. It typically operates on only two AA batteries and are capable enough to comply with the node's application requirements. Unfortunately, it doesn't matter how large is the capacity of the battery or how efficient the protocols are, batteries eventually drains out. It may be a month, year or more 
but with the passage of time, continuous consumption of the limited capacity batteries drains them out eventually. Therefore, it is necessary to replace them to avoid interruption in communication or, in worst case, a complete network outage. In the next subsection, we will explain fixed size batteries deployment and their limitations.

\subsection{Fixed size battery}

Fixed size battery storage is usually referred to as a scenario where battery powered nodes are deployed, but once these attached batteries drain out, it is then practically impossible to replace them. This eventually results in permanent failure of the node and that particular node is then discarded from the network operation. Such scenarios can be typically seen in harsh environmental conditions or hostile military locations, where it is impossible to physically access deployed nodes. The example of one such scenario is described in $[1]$.

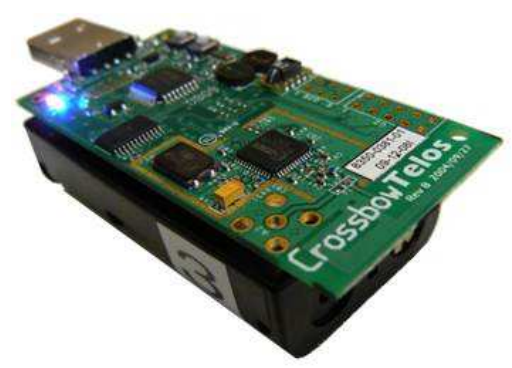

Figure 3: Battery-driven Crossbow's TelosB mote [46]

\subsection{Replaceable fixed size battery}

There are scenarios where accessing nodes for battery replacement is possible, when the attached batteries are drained. The battery replacement can be performed either by humans or robots [4, 47, 48], depending on the conditions where nodes are deployed. For example, there might be human hazardous locations, while robotic replacement is considered as a plausible solution. Most of the current available nodes works on the principle of battery replacement after drainage. Table 1 shows a brief summary of different motes with their respective lifetimes. The maximum achievable lifetime (in hours) is calculated for commercially available nodes, while running only on two typical AA batteries. The sensor motes in Table 1 are tuned to be in active mode, where both the sensing and the communication subsystem simultaneously functions. The ultra low power Telos is just able to achieve a maximum of 241 hours of node operation [49]. This clearly highlights the limitation posed by battery supply regarding the lifetime of most of the common commercially available nodes [45]. Therefore, we recommend supplementing sensor node's attached batteries with alternate energy sources for perpetual lifetime.

We will discuss various battery-driven approaches in the energy consumption section, since most of the previous energy conservation efforts focus is limited to battery-driven sensors. In the subsequent sections, we start with the discussion on how energy provisioning trend is shifting from battery-driven devices to energy harvesting based approaches, and towards most recent, energy transference based energy management approaches.

\section{Energy Harvesting from environment}

Energy provisioning trend for a sensor node is now shifted towards looking for alternate energy sources rather than relying on the limited supply provided by typical batteries [5-8, 29]. Although, some energy management efforts are still concentrated on efficiently utilizing the existing battery-driven model by adapting different techniques, but there is a need to renew energy, specifically, in the form of energy harvesting 


\begin{tabular}{|l|l|l|}
\hline Name of mote & Peak consumption & Expected lifetime \\
\hline Mica & $27.7 \mathrm{~mW}$ & $187 \mathrm{hrs}$ \\
\hline Iris & $24 \mathrm{~mW}$ & $216 \mathrm{hrs}$ \\
\hline Telos & $21.5 \mathrm{~mW}$ & $241 \mathrm{hrs}$ \\
\hline Cricket & $27.7 \mathrm{~mW}$ & $187 \mathrm{hrs}$ \\
\hline Lotus & $66 \mathrm{~mW}$ & $79 \mathrm{hrs}$ \\
\hline
\end{tabular}

Table 1: Lifetime calculation of common commercially available nodes running on $2 \mathrm{x}$ AA batteries

from the environment in order to prolong the sensor network lifetime.

In this section, we shall discuss various efforts utilizing ambient energy along different energy management approaches proposed in this regard. First, we discuss different harvesting opportunities from the environment that can be feasible for sensor networks applications, then we will have an elaborative discussion on state of the art energy management techniques adopted for harvested energy in various research efforts over the past decade.

\subsection{Common energy sources}

The most efficient source of environmental energy is solar power [50] providing most of the energy with very high efficiency. Although, researchers are continuously working on refining the solar cell materials and technology to improve efficiency, but to our recent knowledge, it can harvest energy as efficient as $40 \%$. The total output solar energy depends on the size of the solar panel, but the size constraints associated with sensor node poses limitations towards using a larger sized solar panel. Wind energy can also be utilized as alternate energy source but are infeasible in sensor networks due to the bulky hardware requirement to harvest wind energy as well as its lower order energy harvesting efficiency. Another alternate source uses radio signals for scavenging RF power. Voltage is generated when a time varying RF field passes through an antenna. It follows the principle of mutual inductance by magnetic coupling. Thermoelectric generators are also used to deliver significant power with high temperature. It works on principle of heat transfer based on differences in temperatures of objects. Thermoelectric generators applications are limited to wearable applications or in temperate environments.

Vibration based energy harvesting techniques are widely adapted and several applications such as Automatic Generating System (AGS) and shake-powered flashlights are developed. Other energy scavenging approaches uses piezoelectric materials as their power source. Piezoelectric materials bonded with vibrating structure provides sufficient strain to generate useful energy. The charged capacitors with moving plates can also be utilized as micro-generators but such electrostatic generators must be charged with initial voltage before they start energy generation. The human input can also be utilized as an effective source of power. Therefore, applications based on power from deliberate human input such as cranking, squeezing, shaking, spinning, pumping, pushing and pulling have been developed.

Researchers have presented a shoe-mounted piezoelectric generator to power artificial organs. Various improvements on the shoe mounted energy generation are helpful, specially using electro active polymers made from silicone rubber or soft acrylics, which improves the efficiency of the shoe powered generators by achieving an energy output of about $0.8 \mathrm{~J} / \mathrm{step}$ with heel compression of only $3 \mathrm{~mm}$, yielding $800 \mathrm{~mW}$ of power per shoe at a pace of 2 steps/second [29]. Shoe mounted harvesters can be used in body area networks but their applications are limited to wearable sensor devices.

Among all these sources, solar energy is considered the most appropriate resource for sensor nodes estimated to provide $1.4 \mathrm{~kW} / \mathrm{m}^{2}$ [30]. We evaluated the efficiency of solar energy under different geographical conditions to provide an insight towards the development of energy harvesting aware algorithms and systems for the small sized low powered sensors in WSNs. For this purpose, we analyzed large scale solar radiation 


\begin{tabular}{|l|l|l|l|l|l|l|}
\hline Name & Coordinates & $\begin{array}{l}\text { Mean } \\
\text { Annual } \\
\text { Temp }\end{array}$ & $\begin{array}{l}\text { Data } \\
(\text { days })\end{array}$ & $\begin{array}{l}\text { Mean } \\
\text { Burst } \\
\text { Size }\end{array}$ & $\begin{array}{l}\text { Max } \\
\text { Burst } \\
\text { Size }\end{array}$ & $\begin{array}{l}\text { Mean Daily } \\
\text { Radiation } \\
\left(\mathrm{KW} / \mathrm{m}^{2}\right)\end{array}$ \\
\hline $\begin{array}{l}\text { Tamanrasset, } \\
\text { Algeria }\end{array}$ & $22.78 \mathrm{~N}, 5.52 \mathrm{E}$ & $21.1^{\circ} \mathrm{C}$ & 4744 & 1.32 & 06 & 2.28 \\
\hline $\begin{array}{l}\text { Valentia, Ire- } \\
\text { land }\end{array}$ & $51.93 \mathrm{~N}, 10.25 \mathrm{E}$ & $11.4^{\circ} \mathrm{C}$ & 5752 & 3.85 & 24 & 0.99 \\
\hline $\begin{array}{l}\text { Bondville, IL, } \\
\text { USA }\end{array}$ & $40.06 \mathrm{~N}, 88.37 \mathrm{~W}$ & $11^{\circ} \mathrm{C}$ & 4080 & 1.39 & 05 & 1.47 \\
\hline $\begin{array}{l}\text { Sonnblick, } \\
\text { Austria }\end{array}$ & $47.05 \mathrm{~N}, 12.95 \mathrm{~W}$ & $-04^{\circ} \mathrm{C}$ & 6936 & 2.35 & 18 & 1.45 \\
\hline
\end{tabular}

Table 2: Data set specifications

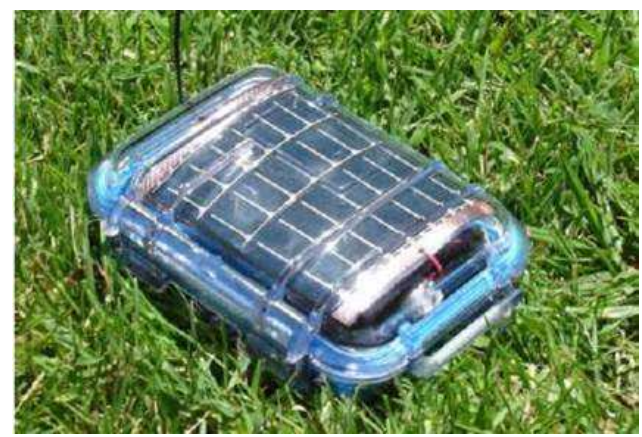

Figure 4: Heliomote [46]

datasets, available at World Radiation Data Center [51]. We selected Tamanrasset (Algeria), Valentia (Ireland), Bondville (Illinois, USA) and Sonnblick (Austria), since these sites provide data for the maximum possible number of consecutive days. As shown in Table 2, the minimum number of days in our analysis is 4080 (Bondville) and the maximum is 6936 for Sonnblick. The sites used in this analysis also vary greatly in solar radiation characteristics. For instance, Sonnblick (Austria) has harsh weather conditions for harvesting solar energy, since it has low average temperature, whereas Tamanrasset (Algeria) possesses better opportunity for harvesting as evident from relatively higher average annual temperature of about $21.1^{\circ} \mathrm{C}$.

We have computed the burst size for each site as the consecutive number of days, in which the daily global solar energy received is less than $0.2 \mathrm{KWh} / \mathrm{m}^{2}$. We chose this threshold considering that a typical sensor node requires around $0.5 \mathrm{~mW}$ power for its active state [45]. Table 2 also shows the mean and maximum burst size for each location. The mean burst size between 1 and 4 days indicates that a variation exists between different sites. The variation of the maximum burst size also indicates the same observation.

The above analysis indicates that the variations in the availability of energy should also be considered while designing harvesting aware algorithms. Additionally, all the above discussed approaches require more attention from the research community in terms of their efficiency. Besides these approaches, there is also a need to further explore alternate energy provision mechanism to overcome the constraints of limited energy source for a sensor node. 


\subsection{Energy management for harvested energy}

Kansal A. [5] have made several research efforts regarding energy management in environmentally powered sensor networks. As the spatially distributed nodes does not have equal harvesting opportunities, therefore, there is a need for energy management based on the available harvested energy at each node. This can be achieved by adjusting the nodes duty cycles according to the availability of the harvested energy. The energy neutral operation is defined, in which nodes utilizes as much energy as available from the environment. The recommendations on selecting an intermediate buffer to store harvested energy are provided with necessary conditions to model the buffer size requirements. The algorithms are proposed to efficiently take into account different environmental changes. In this regard, energy prediction based methods are developed to adjust the power requirements for the available harvested energy. The energy prediction model is based on Exponentially Weighted Moving Average method, in which a day is divided into equal sized time slots. A historical summary of the energy conditions is maintained using the relation: $\bar{x}_{k}=\alpha \bar{x}_{k-1}+(1-\alpha) x_{k}$, where $\alpha$ is the weighting factor, $\bar{x}_{k}$ is the energy generated in a slot, and $\bar{x}_{k-1}$ is the historical average of the previously stored energy.

Kansal A. also proposes an Environmental Energy Harvesting Framework (EEHF) [6] to address the problem of spatio-temporal variations in environmental energy. It is suggested to modify existing load balancing methods, leader election for clustering techniques and energy aware communication. However, some of the challenges include decisions based on knowledge of environmental energy, since the characteristics of environmental energy and the sensing activity cannot be learned by a single node, thus gathering information in a distributed fashion is needed. In addition, they also studied current voltage characteristics of various solar cells and storage devices and their behavior at different environmental variations. Based on the above discussed design considerations, Heliomote [52] is proposed. Heliomote, a solar powered sensor node is shown in Figure 4 is based on Mica2 platform uses Nickel Metal-Hydride (NiMH) batteries as energy storage buffer.

Rahimi M. [7] proposed a model based on energy harvesting such that the network contains mobile nodes. These mobile nodes are able to move freely around the network in search of energy. Once these nodes are charged with sufficient energy, they are then used to charge nodes with critically low energy. The mobile nodes are called energy producers with the assumptions that they can charge themselves from solar energy. The static nodes called as energy consumers, only receive energy from the available mobile nodes. Some issues needs to be addressed such as the available environmental energy pattern may be different than the energy required by the static nodes, in order to avoid energy starvation in the network. The communication overhead for energy queries and replies and the movement overhead of the path taken by the mobile nodes should be taken into account as well. Therefore, the above model is practically demonstrated in a test bed based on robotic mobile nodes and it is shown that up to $40 \%$ of mobile nodes are required to operate the network perpetually. The above model is not feasible in real network scenarios as the additional redundancy by deploying these $40 \%$ additional nodes will degrade the overall efficiency of the network.

In another effort [8], a model is proposed for routing based on the availability of energy harvesting opportunities in the networks. Some modifications are proposed to the existing directed diffusion routing protocol. The objective is to remove dependency on batteries limited power for routing and other necessary operations in a WSN. The network lifetime is increased by utilizing some nodes with solar harvested power for routing purposes. Two solar aware routing protocols are proposed, whose purpose is to perform solar aware routing. Next solar-powered neighbor is selected in routing decision rather than looking for the shortest path neighbor. The standard directed diffusion protocol is made solar aware by adding the solar awareness field and by replacing time stamps with counters. Further, these protocols are experimentally demonstrated and shown to perform better overall network efficiency in term of energy conservation.

Meanwhile, efforts have been made to improve efficiency of nodes with harvesting opportunities. Of those we discussed above, most of the research community focused their attention on only solar energy and more efforts specific to harvesting solar energy can be found vastly in literature as the most feasible alternative 


\begin{tabular}{|c|c|c|c|c|}
\hline Node & Energy Source & Storage Technology & Platform & MPPT \\
\hline Heliomote [52] & $\begin{array}{l}\text { Solar } \\
(3.75 \times 2.5 \text { sq.in } \\
\text { panel })\end{array}$ & $\begin{array}{l}\text { NiMH } \\
\text { mAh) }\end{array}$ & Mica2 & No \\
\hline Ambimax [59] & $\begin{array}{l}\text { Solar } \\
(3.75 \times 2.5 \text { sq.in } \\
\text { panel }), \text { Wind }\end{array}$ & $\begin{array}{llr}\text { Sup-capactor } & (2 \quad \mathrm{x} \\
22 \mathrm{~F}), \quad \text { Li-poly } & \text { Battery } \\
(200 \mathrm{mAh}) & \end{array}$ & Telos & Yes \\
\hline Prometheus [9] & $\begin{array}{l}\text { Solar } \\
(3.23 x 1.45 \text { sq.in } \\
\text { panel })\end{array}$ & $\begin{array}{ll}\text { Sup-capactor } & (2 \quad \mathrm{x} \\
22 \mathrm{~F}), \quad \text { Li-poly } & \text { Battery } \\
(200 \mathrm{mAh}) & \end{array}$ & Telos & No \\
\hline $\begin{array}{l}\text { HydroWatch } \\
{[56]}\end{array}$ & $\begin{array}{l}\text { Solar }(2.3 \times 2.3 \\
\text { sq.in panel })\end{array}$ & $\begin{array}{lll}\text { NiMH } & \text { Battery } & (2500 \\
\text { mAh }) & & \end{array}$ & TelosB & Yes \\
\hline Everlast [60] & $\begin{array}{l}\text { Solar }(2.25 \times 3.75 \\
\text { sq.in panel })\end{array}$ & Sup-capactor $(100 \mathrm{~F})$ & NA & Yes \\
\hline Fleck [57] & $\begin{array}{l}\text { Solar }(4.53 \times 3.35 \\
\text { sq.in panel })\end{array}$ & $\begin{array}{lll}\text { NiMH } & \text { Battery } & (2500 \\
\text { mAh }) & & \end{array}$ & NA & Yes \\
\hline $\begin{array}{l}\text { Solar Biscuit } \\
{[61]}\end{array}$ & $\begin{array}{l}\text { Solar (2x2 sq.in } \\
\text { panel) }\end{array}$ & Sup-capactor (1F) & NA & No \\
\hline Sunflower [62] & $\begin{array}{l}\text { Solar (4-pin } \\
\text { photo diodes) }\end{array}$ & Sup-capactor $(0.2 \mathrm{~F})$ & NA & No \\
\hline
\end{tabular}

Table 3: Energy Harvesting Approaches

energy provision method. One such effort proposes load matching, also called Maximum Power Point Tracking (MPPT) [53, 54]. The aim is to maximize the total energy output of the solar panel by determining the optimal load at run time. Based on the ambient sunlight intensity, the system dynamically adjusts its load to produce the maximum power available for the best system performance [55]. HydroWatch [56], based on low power TelosB platform is a successive effort, which considers Nickel Metal Hydride (NiMH) battery [3] as the storage unit and MPPT for the solar harvest energy. Fleck [57] is another sensor node, which also considers NiMH battery as storage but does not uses MPPT for impedance matching.

Another aspect of environmental powered nodes is to further improve the efficiency by implementing energy management algorithms to reduce power consumption. One such efforts is Prometheus [9]. The energy is harvested using solar panels and the purpose is to hold energy for longer period of time with minimum leakage current and avoiding frequent charge or discharge cycles. Harvested energy is stored using two buffers as shown in the energy management system digram in Figure 5 The primary buffer is a super capacitor [58] as it reduces the frequent charging of battery with the drawback of leakage current at the capacitor, which is mitigated by wiring two super capacitors in series. The Lithium batteries [2] are selected as secondary storage as they can have large number of recharge cycles. Therefore, the system is designed as to first charge the super capacitor. The Lithium battery should be charged only when there is sufficient charge in the super capacitor and it should be used only when solar energy is not available. For this purpose, software-controlled battery charging is introduced to prolong secondary buffer lifetime, which uses Telos's [49] micro-controllers to manage recharge cycles as Telos motes adjusts its duty cycle according to available energy in order to extend network lifetime. Further, the proposed model is then demonstrated in real environment. It is shown that for a certain duty cycle, a mote can achieve near perpetual operation.

AmbiMax [59] is a successive effort, which promises solution for most of the design challenges. It uses a circuitry that is capable of harvesting energy from various sources as shown in Figure 6. AmbiMax also solves the problem associated with MPPT or simply impedance matching at the source and the load. Other design consideration taken into account is to harvest energy from multiple sources, as the previous models were only dependent on solar power availability and does not consider for times when solar power is unavail- 
able. In AmbiMax, a switching regulator is placed between the source and the capacitor. This regulator prevents the capacitor from degrading the performance of the power source by blocking the reverse current flow from the super capacitor to the power source. The proposed model is then implemented and from experimental evaluation it is shown that AmbiMax can charge the super capacitors 12.5 times faster and is able to harvest 3 times more energy under better supply. It performs better at even lower supply levels while other models fails to perform at such conditions.

Everlast [60] is another effort with solar panel along super capacitor and MPPT circuit. It does not considers battery as storage but results in high harvesting efficiency compared to Heliomote and Prometheus due to the MPPT circuit. Other super capacitor based efforts includes Solar Biscuit [61] and Sun Flower [62]. Both these effort does not considers impedance matching using MPPT circuit. All the above discussed efforts are summarized in Table 3. The second column clearly indicates that most of the existing energy harvesting efforts are primarily focused toward utilizing solar energy. Storage technologies used by various efforts can be summarized into three categories, rechargeable batteries, super-capacitors and the hybrid of both batteries and super-capacitors. Heliomote, HydroWatch and Fleck uses NiMH batteries as energy storage buffers for the harvested solar energy. Everlast, Solar Biscuit and Sunflower energy storage units are based on only super-capacitors, while Ambimax and Prometheus utilizes both, super capacitors as well as Lithium polymer batteries as their storage buffers. Ambimax, Hydrowatch, Everlast and Fleck has an advantage of the MPPT circuit which maximizes the solar panel output, thus resulting in higher energy harvesting efficiency.

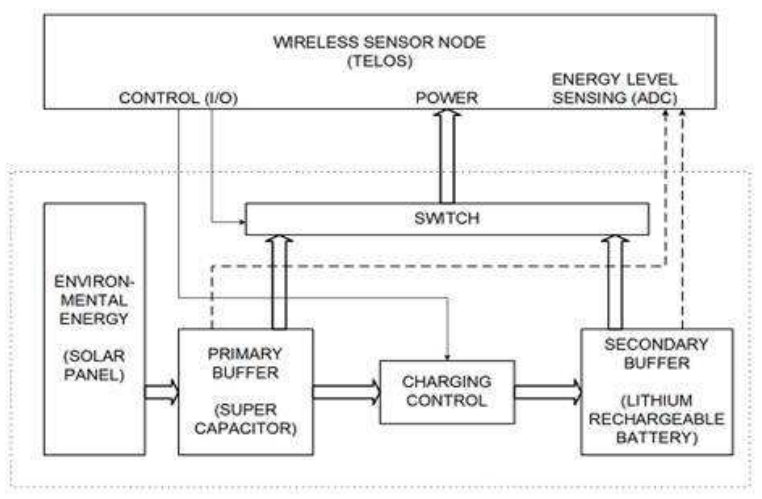

Figure 5: Prometheus energy management system [9]

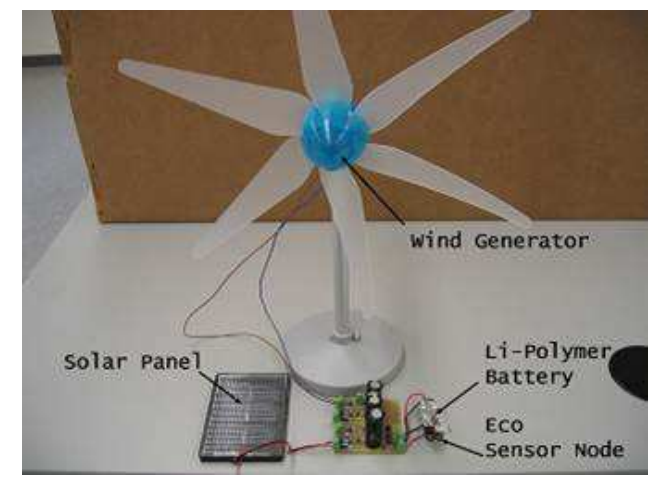

Figure 6: AmbiMax powered by multiple ambient sources [46]

ZebraNet [43] is a sensor network based on single storage lithium ion batteries. Similar to Prometheus, it uses software controlled charging mechanism. It is used for habitat monitoring and for mobility pattern of zebras. The TurtleNet [63] is another sensor network used for habitat monitoring of turtles. Its platform is based on solar energy harvesting using Mica2dot motes. In addition, the Trio [64] is also a sensor network setup based on Telos motes, which uses a hybrid of super capacitors and Lithium ion batteries as storage for the harvested energy. The Shimmer [65] is a super capacitor based sensor network platform used for structural health monitoring. Similarly, VigilNet $[66,67]$ is an example of an integrated energy management system for energy harvesting based sensor network in large scale deployment of a military surveillance system.

Recently, Iannello F. [68] introduced a metric, delivery probability for the performance analysis of energy harvesting Medium Access Protocols (MAC). They considered Time division Multiple Access (TDMA), Framed-Aloha (FA) and Dynamic-Frame Aloha (DFA) for their analysis. Delivery probability metric is used to measure the ability of a sensor's MAC protocol to deliver data to the intended destination. The efficiency of the data collection is investigated using Markov models. 


\begin{tabular}{|l|l|l|l|l|}
\hline Technology & Efficiency & Range & Coverage & Remarks \\
\hline Wired & $90-95 \%$ & $\begin{array}{l}\text { As de- } \\
\text { sired }\end{array}$ & $\begin{array}{l}\text { limited to wires de- } \\
\text { ployment }\end{array}$ & $\begin{array}{l}\text { Static deployment, } \\
\text { Cost }\end{array}$ \\
\hline $\begin{array}{l}\text { Microwave } \\
\text { beam }\end{array}$ & $30-80 \%$ & $>2 \mathrm{Km}$ & Narrow beam & $\begin{array}{l}\text { Potential hazards, } \\
\text { Line of sight required }\end{array}$ \\
\hline $\begin{array}{l}\text { Magnetic } \\
\text { Resonance }\end{array}$ & $45-90 \%$ & $1-2 \mathrm{~m}$ & Omni directional & Limited range \\
\hline $\begin{array}{l}\text { Reflected So- } \\
\text { lar Energy }\end{array}$ & $>90 \%$ & $>1 \mathrm{Km}$ & $\begin{array}{l}\text { Narrow or wide } \\
\text { beam }\end{array}$ & $\begin{array}{l}\text { Daytime only, Line of } \\
\text { sight required }\end{array}$ \\
\hline RF Energy & $70 \%$ & $12-14 \mathrm{~m}$ & Omni directional & Limited range \\
\hline $\begin{array}{l}\text { LASER } \\
\text { beam }\end{array}$ & $10-18 \%$ & $1 \mathrm{Km}$ & Narrow beam & $\begin{array}{l}\text { Potential hazards, } \\
\text { Line of sight required }\end{array}$ \\
\hline
\end{tabular}

Table 4: Possible Energy Transfer Modes

Energy harvesting efforts seems promising toward reducing energy deficiency in sensor networks. However, there exist a requirement for further improvements to cater harsh environmental conditions [1]. In such scenarios, even energy harvesting surrenders, since nodes deployed in a remote region lacks solar energy harvesting opportunities once the attached batteries are drained It is mainly due to the temporal and spatial variation in the availability of solar irradiance. Therefore, it is needed to explore new techniques of charging nodes in the field, which we shall discuss in the next section.

\section{Energy transference}

Energy management in WSNs took a new dimension with the recent advancement of wireless transfer of energy. It overcomes the constraints associated with limited battery power available for sensor nodes, particularly in situations where access to the deployed nodes is almost impossible such as hostile military locations. In this section, we shall discuss some of the various recent efforts carried out in terms of wireless energy transfer in the last few years.

In 2008, David D. L. [10] developed a mobile host, which is capable to wirelessly transfer electrical energy on a $2.4 \mathrm{MHz}$ signal to charge nodes in remote locations. The mobile host is also capable to collect sensing data from the deployed nodes. A test-bed is implemented using a helicopter mounted node. For an assumed distance of two meters, and assuming no losses, attached capacitors are charged in no less than 12 seconds. The transmission and receiving antennas used in the experiment are of the size of the order of $18.7 \times 3$ and $15 \times 15$ inches respectively. Although, this effort explored a new path towards the possibility of charging a node in the field, but using such bulky platform is infeasible in sensor networks due to their limited size and cost requirements. The above effort poses no concern regarding the overhead in terms of size and cost of the sensor node. Increase in the distance between the transmitter and receiver increases the charging duration, reducing the overall efficiency of the system.

Some similar efforts on wireless charging of nodes are proposed [11] using off the shelf devices [31], in order to improve sensor network lifetime. The wireless energy is transferred through electromagnetic waves to sensor nodes equipped with rechargeable batteries. Several experiments were carried out to investigate the effect of distance and location of nodes on the energy transfer. It was observed that when the distance between the transmitter and receiver is increased beyond 12 meters, it takes almost infinite time to charge a particular node. It is also observed that with efficient placement of nodes, the charging time can be substantially reduced. An energy charging cycle aware routing algorithm is proposed by modifying the existing Ad-hoc On Demand Routing (AODV) [69] routing protocols, since typical routing metrics based on shortest path are not applicable in energy harvesting networks. The route request (RREQ) packet includes 
$T_{c h}{ }^{\max }(k)$, the maximum charging time of all nodes traveled on path $k$, and $\eta_{c}{ }^{\max }(k)$, the observed standard deviation of this maximum value. Each node $i$ along the path updates the RREQ with its respective charging time in case its greater than the existing $T_{c h}{ }^{\max }(k)$ field. A delay function $t^{i}{ }_{c h}+\eta^{i}{ }_{c h}$ is also defined as the sum of mean charging time and the its deviation. The destination node selects the minimum charging time path among the available paths.

$$
\begin{aligned}
& \psi=\min \left\{T_{c h}{ }^{\max }(k)\right\}, \forall k, \\
& \psi=\min \left\{\max \left[t_{c h}{ }^{i}\right]\right\} \forall i \in \text { path } \mathrm{k}, \forall k,
\end{aligned}
$$

An optimization framework is proposed to address the trade-offs of the charging and transmission duration, since both occurs in the same frequency band. The base station selects the optimal path, and replies Route Reply (RREP) to the corresponding nodes with the charging time $T_{c h}$ and transmission time $T_{x}$ common to all of the nodes along the path. The optimization framework returns the charging duration $T_{c h}$ and frame length $T_{\text {frame }}=T_{x}+T_{c h}$. Therefore, the source nodes upon receiving the RREP then begins forwarding the packets. The optimization framework is described below:

Given: $\quad L_{\lim }, E S R_{\lim }, N$,

To Find:

$$
T_{\text {ch }}, T_{\text {frame }},
$$

$$
\text { Maximize: } \quad \text { Throughput }=\frac{T_{x} \cdot R}{T_{\text {frame }}},
$$

Subject to:

$$
\begin{array}{r}
\left(E_{\text {rec }}-E_{\text {idle }}\right) \cdot T_{c h}-E_{t x} \cdot T_{x}>0, \\
N\left(T_{c h}+\frac{P+H}{R}\right) \leq L_{\mathrm{lim}}, \\
\frac{1}{E S R_{0}}\left[1-k \cdot t \cdot e^{\left.\frac{-4700}{T+273}\right]}>\frac{1}{E S R_{\mathrm{lim}}},\right. \\
T_{\text {frame }}=T_{x}+T_{c h},
\end{array}
$$

For $N$ number of nodes in the path, throughput is defined as the ratio of the number of bits sent during $T_{x}$ to the frame length $T_{\text {frame }}$. To maximize the throughput, the constraints are also defined, where $E_{\text {rec }}$ is the energy receiving rate from the wireless charger and $E_{i d l e}$ is the sensor node's idle energy during charging time. $E_{t x}$ is defined as the rate, at which a sensor node losses energy during transmission. Similarly, $P$ is the packet size, $H$ is the header size and $R$ is defined as the sending rate of the data at the $N$ hop route. The constraints are explained below:

- The first constraint in (4) ensures that the sensor is alive after each frame duration. The sensor expends $E_{\text {idle }}$ during its charging time while it receives energy at the rate $E_{r e c}$ from the wireless transmitter in time $T_{c h}$. It also loses energy $E_{t x}$ during transmission as mentioned above, thus, the residual energy should at be at least greater than 0 .

- The second constraint in (5) states that the end-to-end packet latency for the $N$ hop route should be less than a pre-decided limit $L_{\mathrm{lim}}$. In the worst case, a sensor may experience a delay equal to the charging time $T_{c h}$, where no data can be sent and the transmission delay, given as the ratio of the packet size $P$ with the header size $H$ and the sending rate $R$. 
- The Equivalent Series Resistance (ESR) in (6) is a metric which determines the quality of capacitor operation. The capacitors are considered dysfunctional once the end-end latency limit $L_{\text {lim }}$ is exceeded. $L_{\lim }$ and the capacitor quality metric $E S R_{\lim }$ are dependent on the application requirements. The $T$ is the absolute temperature in Kelvin, at which the capacitor operates. Similarly, $t$ is the operational time and $k$ is a design constant.

- The constraint in (7) provides the relationship between the charging and transmission times and the the frame time.

The performance evaluation highlights the following situations:

1. When $L_{\text {lim }}$ is too small, either the node will not be able to charge enough or it may not be alive after each transmission.

2. By considering values of the charging time lower than the optimal derived $T_{c h}$, the network throughput is substantially increased. However, the rate at which the throughput increases exhibits a non-linear behavior, thus hinting that for sudden high bandwidth needs, decreasing the recharging time (thereby increasing the transmission time) will not incur a proportionally high degradation of lifetime. Moreover, we also observed that different packet sizes do not significantly impact the performance.

Powercast Corporation provides off the shelf devices [31] which are used in the above work for wireless energy transfer. These devices supports RF energy based energy transfer. They do not increase the sensor size substantially, but to address the constraint imposed by distance effect, further efforts are needed.

Some recent progress $[70,71]$ started to further improve the network lifetime with the help of wireless energy transfer by optimizing the charging process for the nodes deployed in the field. In [12], a mobile Wireless Charging Vehicle (WCV) is introduced. It periodically visits and charges the batteries of the nodes deployed in the field. The energy is transferred to the nodes through magnetic resonance based technology known as Witricity [33]. In [70], It is assumed that the WCV charges itself from a service station with virtually infinite amount of energy as shown in Figure 7. It then travels the optimized path through the network of nodes deployed moving along the shortest Hamiltonian cycle in order to efficiently utilize its charging cycle, thus increasing its vacation time. The vacation time is referred as the time spent by the WCV at the service station. The authors also introduced a renewable cycle, in which the amount of charged energy at a sensor node in a specific time duration must be equal to the amount of energy consumed in the cycle. Similar work is extended to incorporate the multi- node case in [14]. Similarly, in [71], each sensor node deployed in the field optimally tunes its data rate based on the current energy replenishment status. The WCV, named as SenCar, dynamically adjusts its visiting time for each node as well.

The above work is more specific on theoretically optimizing the path of the WCV rather than exploring a detailed case of energy transference. Other energy charging efforts based on wireless energy transfer are proposed in $[72,73]$. Although, wireless energy transfer aim to reduce energy deficiency, but there is still a need to further explore wireless energy transfer using different technologies.

In a recent effort [74], it has been derived to improve network lifetime by wirelessly charging nodes using multi-hop energy transfer. The magnetic resonance based technology, Witricity is used and it is shown that by reducing the radii of coils, while increasing the number of turns, the transferred energy can be increased substantially. Three different energy transfer techniques are proposed, namely, Store and forward, direct flow, and hybrid schemes. Store and forward based approach, in which energy received at an intermediate nodes from previous node is first stored in a buffer, then forwarded to the next hop node when the battery is fully charged at the cost of additional charging and discharging losses at each hop. In direct flow, the intermediate node directly transmits the received energy to the next node without storing it in its buffer, thus reducing the charging and discharging losses except at the last node, which will eventually use a battery to store the energy it receives. The Hybrid technique uses the combination of both, store and forward technique as well as direct transmission. Hybrid schemes always performs better than the previous two 


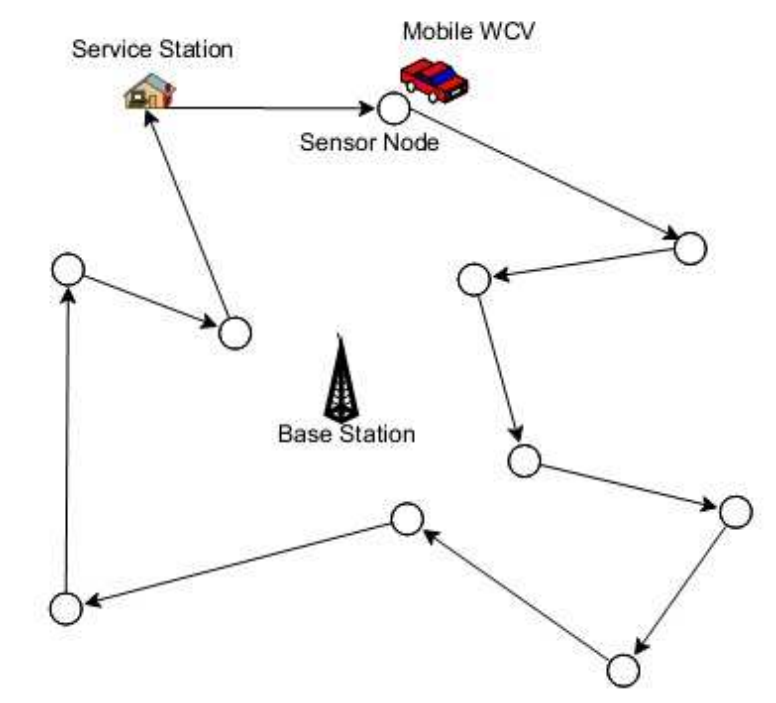

Figure 7: Wireless charging vehicle (WCV) travels along optimal path to charge sensors deployed in the field [12]

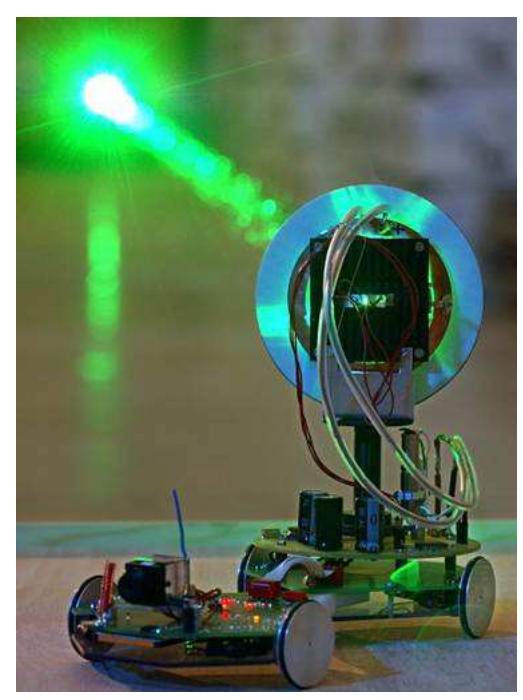

Figure 8: An example of LASER power transfer [15]

techniques for a large number of total hops.

An example of LASER reflection based energy transfer is demonstrated in [15] as shown in Figure 8. Table 4 summarizes various possible energy transference technologies. The major technologies used by most of existing efforts are RF based and magnetic resonance based energy transfer. It is mainly due to their mobility, high conversion efficiency and property to provide omni-directional coverage. These technologies lacks efficiency in terms of distance, since energy transference is limited by distance constraint. The nodes need to be deployed in close proximity in order to efficiently transfer energy. Therefore, these technologies requires further improvements, specifically in sparse sensor network deployment, where nodes might require to transmit energy to a node far more than the maximum range supported by any of these existing transference technologies. Instead on relying on magnetic resonance and RF energy, there is a need to further explore alternate choices such as microwave power beaming and reflection based technologies, which provides improvement in term of distance. However, there is a trade off regarding coverage since these technologies are constrained by narrow beaming and the requirement of line of sight poses a challenge as well.

\section{Energy consumption based energy management}

Wireless Sensor Networks have gained lot of attention in the last decade due to their low power consumption nodes and mobility. But researchers are still not satisfied and further efforts are in progress in order to improve the power consumption by increasing the efficiency of the battery-driven sensors. In this section, we shall discuss different approaches adopted by the research community to improve battery-driven device's efficiency aiming to keep the network perpetual. Rather than discussing each protocol separately, we shall provide a general idea of the key approaches, which are adapted by different protocols. We categorize our discussion into three major subsections in order to clearly identify characteristics of each scheme.

\subsection{Duty Cycling}

Duty cycling is considered as the most effective way of improving network's lifetime. Several algorithms are proposed, in which nodes adjust their duty cycle by alternating between sleep and wakeup modes to 
reduce battery consumption. The idea is to set the node in a low power mode when there is no data communication in progress. In this way, energy wastage is avoided as the nodes only wakes up when there is a need of radio transmission or reception. Such algorithms can prolong the overall network lifetime by utilizing the battery only when they are awake. Duty cycle based algorithms can be further categorized as Topology control protocols, Sleep/wake-up protocols and MAC protocols with low duty-cycles.

Topology control Protocols refers to schemes that adapts dynamic network topology in accordance to the application requirements. The aim is to set some nodes in sleep mode while keeping the network operational, hence prolonging network lifetime. Such protocols can be further divided as location driven and connectivity driven. In Location driven approaches, nodes are set to sleep or wake-up mode on the basis of their location. The location of the nodes are assumed to be known so that nodes can coordinate with each other to decide, which node in a particular area should be turned off, while not compromising coverage of that particular area.

Topology control schemes aim to reduce the topology and maintain it for topology conservation. Most of the efforts in this domain lie in the area of Connected Dominating Set (CDS) or backbone, which aims to form a reduced topology working on behalf of other nodes in the network. In this area, the authors proposed CDS Rule $\mathrm{K}$ algorithm [75] that uses marking and pruning rules for exchanging neighbors list among a set of nodes. In CDS Rule K, a node remains marked as long as there is at least a pair of unconnected nodes in its neighbors; it is unmarked when it finds that all its neighbors are covered with high priority. Similarly, Energy Efficient CDS (EECDS) algorithm is proposed in [76], which also follows a two phase topology control scheme in order to form a connected dominating set based on coordinated reconstruction mechanism to prolong network lifetime and balance energy consumption. On the other hand, the authors in [77, 78] propose A3 and A1 algorithm which constructs a backbone or a CDS in a single phase while in [79], the authors propose Poly algorithm, which provides reliability in addition to energy efficiency by constructing a backbone in a single phase.

For evaluation of topology control algorithms, we used Attaraya simulator [80] that has been specifically designed for WSNs. The Atarraya underlying features provide many advantages which includes, different energy and communication models, energy and node location distribution resources that can adapted according to the requirement in the simulations. On the other hand, the performance of the algorithms was evaluated under two metrics namely energy overhead and residual energy. The former shows the overhead associated or the energy consumed during the exchange of the messages, while the latter shows the remaining energy at the end of Topology Control operation. For evaluation of the algorithms under discussion, the nodes were distributed in an area of $600 \mathrm{~m} \times 600 \mathrm{~m}$ while varying the node density from 50 to 250 nodes. Similarly, the algorithms were also evaluated for indoor Grid H-V and H-V-D topologies. In Grid H-V and $\mathrm{H}-\mathrm{V}-\mathrm{D}$, nodes communicate with their horizontal, vertical and diagonal neighbors depending upon the topology deployed. The transmission radius and initial energy level of each node are set to $42 \mathrm{~m}$ and $1 \mathrm{~J}$, respectively. The actuation energy equals $50 \mathrm{~nJ} /$ bit while the communication energy is $100 \mathrm{PJ} / \mathrm{bit} / \mathrm{m}^{2}$. All the results were averaged over 100 simulations runs and the nodes energy distribution follows a uniform process while the node location distribution follows a random process.

Figure 9 demonstrate the energy overhead incurred by different topology control algorithms under Grid $\mathrm{H}-\mathrm{V}$ and Grid H-V-D topologies and under varying node density. The results demonstrate that the schemes using two phase backbone topology construction mechanism incur more energy overhead due to the use of large number of messages, while the schemes constructing backbone in a single phase incur less energy overhead. Similarly, the residual energy is also remains high for schemes with single phase mechanism for the same reasons mentioned earlier and therefore provides better energy efficiency as shown in Figure 10.

The authors in [81] introduced hyper-graph theory based topology control algorithm to replace simple graphs in large scale WSNs. Simple graph theory based algorithms results in high computational complexity and usually requires large solution space to manage large scale WSNs due to their small granularity. The transmission paths computed using traditional graph theory techniques provide lower fault tolerance 

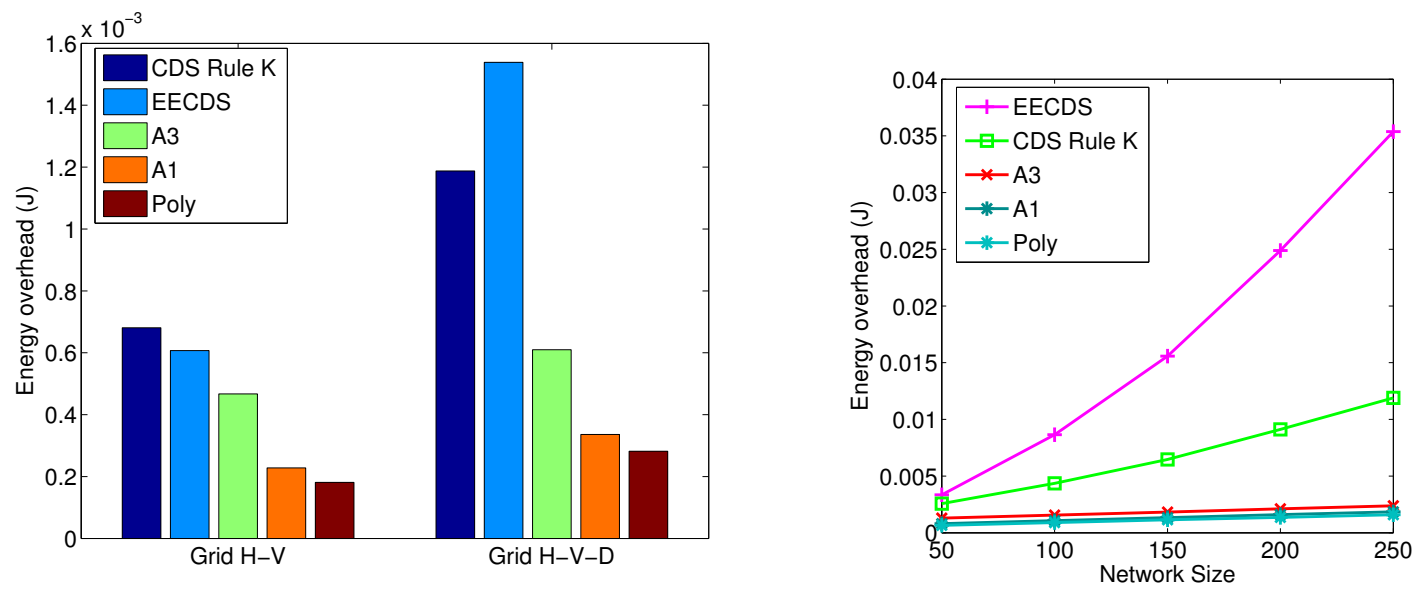

Figure 9: Energy overhead comparison under Grid H-V and H-V-D topologies and under varying node density.
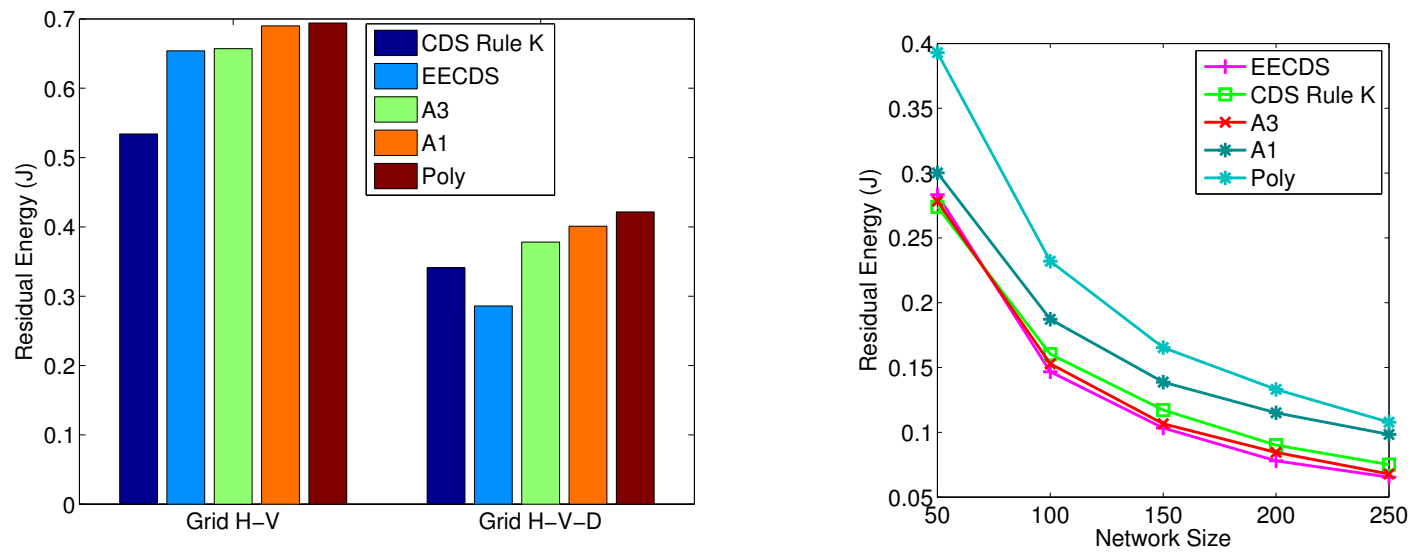

Figure 10: Residual Energy comparison under Grid H-V and H-V-D topologies and under varying node density.

due to unattended sensor nodes operations and unreliable wireless transmission channels. To maintain the connectivity of a delivery path, lots of control message to are required. Such control messages uses more bandwidth with increased energy consumption.

A hyper graph based tool, namely Spanning Hyper-graph Tree (SHT) is proposed to generalize the high connectivity in wireless self-organized networks into concise and robust hyper-graph infrastructure. SHT is the first hyper graph model where nodes and connected edges among them are generalized as hyper-edges. With the growth of hyper-edges, as the minimum computing unit, fewer extra packets are used which substantially reduces the energy consumption. SHT also ensures time synchronization between nodes to reduce complexity. The issue of limited solution space is addressed by using variable scale hyper edges. Similarly, the use of mutual back-up delivery paths in a single hype edge improves the fault tolerance capability. Comparison results with simple flooding, Directed Diffusion [82], EADD [83] and Enhanced Fault Tolerant AODV (ENFAT AODV) [84] shows the improvement of SHT in terms of its average dissipated energy, average latency and the packets loss ratio.

Besides topology control algorithms, energy efficient routing algorithms such as AODV, Directed Diffu- 
sion, SPEED [85] and Reliable Energy Aware Routing protocol (REAR) [86] also ensure minimum energy consumption at relay nodes. Recently research focus is shifted toward energy efficient fault tolerant routing algorithms. One such algorithm DLS (Dynamic local stitching) is proposed recently in [87]. DLS aims to repair broken transmission paths in WSN, specially, in harsh environments comprising unattended sensor nodes with unreliable wireless transmission channels. Unlike typical routing algorithms such as AODV, ENFAT-AODV, Directed Diffusion, SPEED and REAR which reroute the entire paths. DLS only repairs broken fragments of the original path, thus minimizing energy consumption as well as the recovery delay. Figure 11 compares nodes energy consumption using different routing algorithms with increasing fault tolerance percentage from $5 \%$ to $30 \%$ of faulty nodes in the transmission path. DLS, with its fewer overhead packets rectifies broken path with a lower latency compared to other routing algorithms.

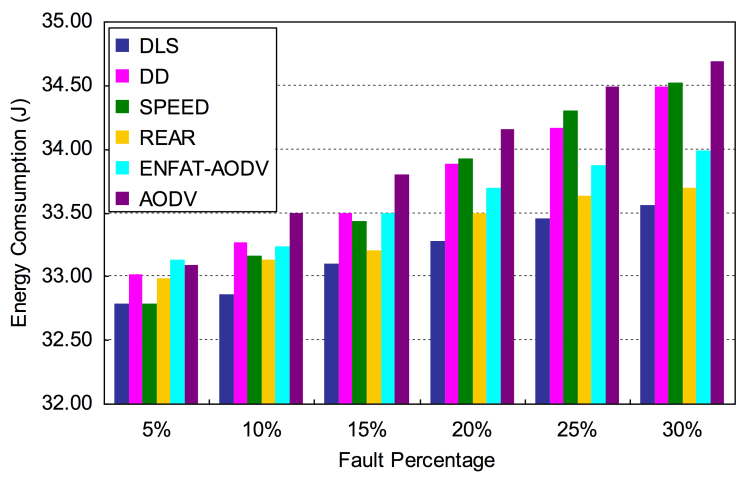

Figure 11: Energy consumption comparison of different routing schemes with respect to percentage of faulty nodes [87]

Geographical Adaptive Fidelity (GAF) [17] and Geographical Random Forwarding (GeRaF) [18] are typical examples based on location driven approaches. The connectivity driven approach on the other hand, are generally more preferred protocols. The nodes can be set to be in a sleep or wake up mode, while keeping the network well connected and scalable. The protocols such as Span [19] and Adaptive Self Configuring sEnsor Networks Topologies (ASCENT) [20] are examples of efforts implementing such approach. These efforts although demonstrated that topology control protocols provide better efficiency in terms of increasing network lifetime but efforts are still required to couple such protocols with other energy conservation protocols for perpetual network operation.

Duty cycling protocols can also be further classified on the basis of power management by adapting different sleep and wake-up protocols or MAC protocols having low duty cycles. For better understanding, sleep and wake-up protocols are further divided into on-demand, scheduled rendezvous and asynchronous protocols. On-demand protocols are used in event driven scenarios, where nodes should only wake-up when they are needed for communication. Typical Examples of such schemes are Sparse Topology and Energy Management (STEM), Pipeline Tone Wake-up (PTW) and Radio Triggered sleep wake-up schemes [88-90]. The issue related to informing a sleeping node to wake-up is addressed by using multiple radios. A low rate and low power radio is used for signaling and a more power consuming radio is used for data communications. It seems to be ideal protocol but radio triggered wake-up scheme is not a feasible solution. It can only be applied in situations where nodes are in close proximity to each other. In addition, using a second radio seems to be an unrealistic approach. In scheduled rendezvous, nodes wake-up according to a schedule and remain active for a short period and then enter sleep mode till the next rendezvous time arrives. Such protocols are convenient, although, synchronization is required between the nodes. Such schemes also poses a drawback in terms of additional synchronization overhead. In asynchronous protocols, a node can wake-up independently of others and can still be able to communicate with its neighbors. It is easier to implement and ensures network connectivity even in highly dynamic conditions. Asynchronous protocols are said to be 


\begin{tabular}{|c|c|c|}
\hline TDMA based & Contention based & Hybrid \\
\hline TRAMA [21] & B-MAC [24] & Zebra MAC [27] \\
FLAMA [22] & S-MAC [25] & HYMAC [91] \\
L-MAC [23] & D-MAC [26] & \\
\hline
\end{tabular}

Table 5: Low power MAC schemes

less energy efficient, therefore, consequently resulting in higher duty cycles than synchronous nodes.

An alternative duty cycling approach is by applying MAC protocols with low power consumption. These can be classified as Time Division Multiple Access (TDMA) based, contention based and hybrid MAC protocols as shown in Table 5 .

In TDMA based protocols, nodes duty cycle is enabled only when channel access is required. A fixed time slot is assigned to each node and the energy consumed is reduced to only the respective time slot. The protocols such as Traffic-Adaptive MAC Protocol (TRAMA) [21], Flow Aware Medium Acces (FLAMA) [22] and Lightweight Medium Access Control (L-MAC) [23] are the most common ones adapting such schemes. The TDMA based schemes provides efficiency in terms of energy consumption, since nodes turn on their radios only in their own allotted time slot. These protocols provides limited flexibility and are generally scalable, however, they often requires tight synchronization and are very sensitive to interference. These protocols perform worse than contention based protocols in low traffic conditions and are therefore, rarely used in WSNs.

The contention based protocols on the other hand, achieves duty cycling by integrating medium access functionality with sleep or wake-up process. The most common schemes based on this principle are Berkeley MAC (B-MAC), Sensor MAC (S-MAC) and Data gathering MAC (D-MAC) [24] [25] [26]. These protocols are robust and scalable and maintains lower delay than TDMA based protocols, however, they results in high energy consumption due to contention and collisions.

The hybrid schemes refers to algorithms combining properties from both, TDMA based and contention based schemes. It behave as contention based scheme when the level of contention between nodes is low, and then switches to TDMA based scheme when the level of contention is higher. These are complex schemes and are feasible only in situations where high numbers of nodes are deployed. The Zebra MAC (Z-MAC) [27] and Hybrid MAC (HYMAC) [91] are popular hybrid scheme combining strengths of TDMA based and Carrier Sense Multiple Access (CSMA) based schemes.

Dynamic duty cycling is also applied by few researchers for harvesting enabled sensors [92]. The energy allocation to the application is based on the availability of daily harvested energy. Kansal A. provided an adaptive duty cycling scheme for the energy harvesting sensor node in [34]. Moreover, Dynamic Power Management (DPM) algorithms can also be used to efficiently manage energy for a sensor node. In DPM, the sensor node is turned off when there is no sensing activity and triggered in case of occurrence of any event [93]. Such algorithms mostly suffer from the overhead of sleep state transitions, specifically, in storage and retrieval of the sensor processing state during switching. Similarly, Dynamic Voltage-Frequency Scaling (DVFS) can also be adapted to manage the node energy consumption [94, 95]. DVFS schemes allow the node to operate at the maximum processing speed if the stored energy is sufficient, otherwise, the system reduces the execution of sensing tasks in order to conserve energy [96]. The efficiency of such schemes to save energy depends on the application requirements for task execution. Energy conservation for longer network operation is only possible when sensing requests by the application are less frequent. 


\begin{tabular}{|c|c|c|}
\hline \multicolumn{2}{|c|}{ Type of Scheme } & Example Protocols \\
\hline $\begin{array}{l}\text { Duty- } \\
\text { cycling }\end{array}$ & $\begin{array}{l}\text { Topology Control } \\
\text { Sleep/Wake-up } \\
\text { Low duty-cycle MAC }\end{array}$ & $\begin{array}{l}\text { GAF [17], GeRaF [18], Span [19], ASCENT [20] } \\
\text { STEM [88], PTW [89], Radio Triggered } \\
\text { Sleep/wake-up [90] } \\
\text { TRAMA [21], FLAMA [22], LMAC [23], BMAC } \\
\text { [24], SMAC [25], DMAC [26], ZMAC [27], HY- } \\
\text { MAC [91] }\end{array}$ \\
\hline $\begin{array}{l}\text { Data- } \\
\text { driven }\end{array}$ & $\begin{array}{l}\text { Data Prediction } \\
\text { Energy Efficient Data } \\
\text { Acquisition }\end{array}$ & $\begin{array}{l}\text { Ken [97], PAQ [98], SAF [99], PREMON [37], } \\
\text { EEDC [28] } \\
\text { BBQ [38], ASAP [100], USAC [101] }\end{array}$ \\
\hline $\begin{array}{l}\text { Mobility- } \\
\text { based }\end{array}$ & $\begin{array}{l}\text { Mobile Sink } \\
\text { Mobile Relay }\end{array}$ & $\begin{array}{l}\text { GMRE [39], TTDD [40], SEAD [102] } \\
\text { Message Ferrying [41], Data-MULE [42], Ze- } \\
\text { braNet [43] }\end{array}$ \\
\hline
\end{tabular}

Table 6: Energy Consumption based Energy Management Schemes

\subsection{Data Driven approaches}

Data driven approaches are generally focused to reduce the amount of sampled data while keeping sensing accuracy within the acceptable level. Such approaches can be classified as data reduction schemes and energy efficient data acquisition. The data reduction schemes address the case of unneeded samples. The data prediction is a further classification of data reduction schemes, which are focused on building an abstraction of the sensed data, or in other words, a model for future data prediction.

The data prediction schemes can be further divided into stochastic approaches, time series forecasting and algorithmic approaches. The stochastic approaches works on the principle of stochastic characterization of the phenomena as proposed in the Ken solution [97]. Such protocols are involved in high-level computations such as aggregating, with the expense of high computational costs. These approaches are feasible in situations where powerful sensor nodes are available in the network, thus requires larger battery size. In time series forecasting, set of historical values are obtained by periodical sampling, which are then used to predict a future value in the same series. The most common techniques using these approaches are Moving average (MA), Auto-regressive (AR) or Auto-regressive moving average (ARMA) methods. These schemes are simpler and lightweight in implementation and provides satisfactory results in terms of accuracy. Examples of such approaches are Probabilistic Adaptable Query system (PAQ) and Similarity based Adaptive Framework (SAF) [98, 99]. In algorithmic approaches, heuristic or state transition model describing sensed phenomena are used. Typical examples of such approaches are Prediction based Monitoring in Sensor Networks (PREMON) and Energy Efficient Data Collection (EEDC) [28, 37]. These techniques are considered case by case as they are more application specific schemes. In addition, few energy prediction algorithms and dynamic duty cycling based on the available harvested energy are proposed in [35, 36, 103-106].

Energy efficient data acquisition protocols are more focused towards reducing energy consumption of the node sensing subsystem. Such protocols assume that greater amount of energy is consumed by the sensing sub system of the node than the communication subsystem. These schemes are further divided as adaptive sampling, hierarchical sampling and model based active sampling. In adaptive sampling, the main focus is to reduce the amount of data to be acquired from the transducer based on either spatial or temporal correlation between data. These schemes are more general and efficient, and mostly implemented in a centralized fashion, thus requiring high computations. In hierarchical sampling, different types of sensors are installed on nodes. These schemes are more energy efficient, but are more application specific. However, the cost associated with the extra transceiver can be considered as a drawback of such schemes. The model based approaches are similar to data prediction schemes. The goal is to reduce the number of data samples by using computed models and saving energy through data acquisition. Protocols such as Barbie-Q (BBQ) 
[38], Adaptive Sampling Approach to Data Collection (ASAP) [100], and Utility Based Sensing and Communication (USAC) [101] are based on such schemes.

\subsection{Mobility based approaches}

Mobility based energy conservation can be achieved by considering few mobile nodes in the network. These mobile nodes can be of two types, based on their behavior. They can either be part of the network infrastructure in which their mobility is fully controllable or generally a robotized one. Such nodes may follow a predictable pattern of mobility. On the other hand, they can be part of the environment, in which nodes mobility is uncontrollable and unpredictable. However, in some cases they might follow a mobility pattern that is neither predictable nor random in general [16].

Mobility based algorithms can be further divided in two categories. A mobile sink based approach, in which a mobile sink is used to collect data from source nodes in the field in order to increase network lifetime. It is shown that using mobile sinks nodes can improve networks lifetime by 5 to 10 times than using static sink nodes. However, the possibility of latency associated with the data arrival at the sink node should be taken into consideration. Some examples of such approaches are Greedy Maximum Residual Energy (GMRE) [39], Two Tier Data Dissemination (TTDD) [40] and Scalable Energy-efficient Asynchronous Dissemination (SEAD) [102] protocol. In a mobile relay based approach, message ferries are used for data collection from source nodes [41]. These message ferries moves in the field to collect data, carry the stored data and forward it to the destination node. Mobile relays have almost similar functionality as in data Mobile Ubiquitous LAN Extension (MULE) approaches [42], where the vehicles periodically visit a network to collect data. However, some issues needs to be addressed such as the sensors have to be continuously in wake-up mode while waiting for the MULE to arrive for data collection. The transmission schedule need to be defined to address the issue of the amount of time a MULE has to wait for data coming from the static nodes, and vice versa, when a sensor should transmit gathered data to the mobile element. Typical example of mobility based approaches is ZebraNet [43].

Energy efficient network operation is also possible by defining several application layer protocols. Typical application layer protocols can be categorized into three types; Sensor Management Protocols (SMP), Task Assignment and Data Advertisement protocols (TADAP) and Sensor Query and Dissemination protocols (SQDDP).

- Sensor Management Protocols (SMP) are used by network administrators to configure nodes to perform various tasks. These protocols can be used to introduce rules regarding data aggregation [107], time synchronization [108], sensor movements, clustering, authentication and key distribution [109, 110].

- Task Assignment and Data Advertisement protocols (TADAP) are used to handle users interests and sensor node advertisements. The users query for the sensing data they are interested and the corresponding sensor nodes advertise the requested data. TADAP provides the user software with efficient interfaces for interest dissemination which also supports energy efficient lower-level operations $[111,112]$.

- Sensor Query and Dissemination protocols (SQDDP) are designed for attribute or location based sensor query. Typical example of such query could be for the location of all the nodes sensing temperature higher than a certain threshold, where the threshold can be defined by the user. These protocols are helpful in ensuring messages exchange between the user and the sensor deployed in the field under different conditions [113].

\subsection{Discussion}

Energy consumption based energy management schemes are summarized in Table 6. Duty cycling based energy management approaches are proposed in majority of energy conservation schemes as shown in Table 
6. Besides duty cycling, state of the art Data driven schemes and Mobility based schemes are also identified. Among all the energy conservation schemes, topology control schemes which are based on duty cycling are the most recent and provide better energy efficiency and longer network lifetime. However, most of the current efforts are focused only towards energy conservation based on efficient energy consumption. Energyefficient design of a sensor hardware, software, algorithms and protocols have served well, but they eventually surrender when the attached batteries are drained. For instance, an energy-efficient protocol which relies on duty cycling of spatio-temporal sensing activities may result in application performance degradation for the sake of longer network lifetime. Therefore, our analysis reveals that it is better to renew energy rather than relying on fixed energy already coupled with the node at the time of network deployment. The high level taxonomy provides an insight for the research community to consider the trend toward energy replenishment using alternate sources while catering for energy efficiency in WSNs.

\section{Conclusions/Future Work}

This paper presented a comprehensive survey on different energy management schemes in WSNs. Theses schemes are classified into two categories, energy provision based and energy consumption based. Energy provision approaches studies the energy source characteristics and develop algorithms depending on the energy availability to the sensor. We categorized such schemes as battery driven, energy harvesting, and energy transference based schemes. On the contrary, energy consumption based schemes refers to the algorithms and protocols that does not take into account the node's energy source. This survey is more focused towards identifying the potential of various alternate energy sources and the different efforts on their efficient utilization. A network wide energy efficient protocol can better manage its operation while taking into account the nodes' supply and consumption. Therefore, we recommend to the research community to consider both, the energy supply as well as the energy consumption in parallel while designing an energy efficient algorithm.

Alternative energy sources from ambient environment and wireless transference based algorithms still require further improvements. Existing sources requires improvement regarding their energy harvesting efficiencies as well considering the possibility of exploring new sources. A hybrid technique comprising all the three existing sources (batteries, ambient environment, and wireless transfer) can also be considered to increase the network lifetime. However, it is still an open research issue and lots of efforts are required towards the realization of a perpetual WSN.

\section{References}

[1] I. Talzi, A. Hasler, S. Gruber, C. Tschudin, Permasense: investigating permafrost with a wsn in the swiss alps, in: Proceedings of the 4th workshop on Embedded networked sensors, ACM, 2007, pp. 8-12.

[2] B. Scrosati, R. J. Neat, Lithium polymer batteries, in: Applications of Electroactive Polymers, Springer, 1993, pp. $182-222$.

[3] Nickel metal hydride battery- http://www.batteryspace.com/nimhpacks24-48v.aspx.

[4] B. Tong, G. Wang, W. Zhang, C. Wang, Node reclamation and replacement for long-lived sensor networks, in: Sensor, Mesh and Ad Hoc Communications and Networks, 2009. SECON'09. 6th Annual IEEE Communications Society Conference on, IEEE, 2009, pp. 1-9.

[5] A. Kansal, J. Hsu, S. Zahedi, M. Srivastava, Power management in energy harvesting sensor networks, ACM Transactions on Embedded Computing Systems (TECS) 6 (4) (2007) 32.

[6] A. Kansal, M. Srivastava, An environmental energy harvesting framework for sensor networks, in: Low Power Electronics and Design, 2003. ISLPED'03. Proceedings of the 2003 International Symposium on, IEEE, 2003, pp. 481-486.

[7] M. Rahimi, H. Shah, G. Sukhatme, J. Heideman, D. Estrin, Studying the feasibility of energy harvesting in a mobile sensor network, in: Robotics and Automation, 2003. Proceedings. ICRA'03. IEEE International Conference on, Vol. 1, IEEE, 2003, pp. 19-24.

[8] T. Voigt, H. Ritter, J. Schiller, Utilizing solar power in wireless sensor networks, in: Local Computer Networks, 2003. LCN'03. Proceedings. 28th Annual IEEE International Conference on, IEEE, 2003, pp. 416-422.

[9] X. Jiang, J. Polastre, D. Culler, Perpetual environmentally powered sensor networks, in: Information Processing in Sensor Networks, 2005. IPSN 2005. Fourth International Symposium on, IEEE, 2005, pp. 463-468.

[10] Mascarenas, david d. l.(2008). "mobile host" wireless sensor networks : a new sensor network paradigm for structural health monitoring applications. uc san diego: b6636138. retrieved from: http://escholarship.org/uc/item/27k5c57r. 
[11] R. Doost, K. Chowdhury, M. Di Felice, Routing and link layer protocol design for sensor networks with wireless energy transfer, in: GLOBECOM 2010, 2010 IEEE Global Telecommunications Conference, IEEE, 2010 , pp. 1-5.

[12] L. Xie, Y. Shi, Y. T. Hou, H. D. Sherali, Making sensor networks immortal: An energy-renewal approach with wireless power transfer, IEEE/ACM Transactions on Networking (TON) 20 (6) (2012) 1748-1761.

[13] A. Kurs, A. Karalis, R. Moffatt, J. Joannopoulos, P. Fisher, M. Soljačić, Wireless power transfer via strongly coupled magnetic resonances, science 317 (5834) (2007) 83.

[14] L. Xie, Y. Shi, Y. T. Hou, W. Lou, H. D. Sherali, S. F. Midkiff, On renewable sensor networks with wireless energy transfer: The multi-node case, in: Sensor, Mesh and Ad Hoc Communications and Networks (SECON), 20129 th Annual IEEE Communications Society Conference on, IEEE, 2012, pp. 10-18.

[15] L. Summerer, O. Purcell, Concepts for wireless energy transmission via laser, Europeans Space Agency (ESA)-Advanced Concepts Team.

[16] G. Anastasi, M. Conti, M. Di Francesco, A. Passarella, Energy conservation in wireless sensor networks: A survey, Ad Hoc Networks 7 (3) (2009) 537-568.

[17] Y. Xu, J. Heidemann, D. Estrin, Geography-informed energy conservation for ad hoc routing, in: Proceedings of the 7th annual international conference on Mobile computing and networking, ACM, 2001, pp. 70-84.

[18] M. Zorzi, R. Rao, Geographic random forwarding (geraf) for ad hoc and sensor networks: multihop performance, Mobile Computing, IEEE Transactions on 2 (4) (2003) 337-348.

[19] B. Chen, K. Jamieson, H. Balakrishnan, R. Morris, Span: An energy-efficient coordination algorithm for topology maintenance in ad hoc wireless networks, Wireless Networks 8 (5) (2002) 481-494.

[20] A. Cerpa, D. Estrin, Ascent: Adaptive self-configuring sensor networks topologies, mobile computing, IEEE transactions on 3 (3) (2004) 272-285.

[21] V. Rajendran, K. Obraczka, J. Garcia-Luna-Aceves, Energy-efficient, collision-free medium access control for wireless sensor networks, Wireless Networks 12 (1) (2006) 63-78.

[22] V. Rajendran, J. Garcia-Luna-Aveces, K. Obraczka, Energy-efficient, application-aware medium access for sensor networks, in: Mobile Adhoc and Sensor Systems Conference, 2005. IEEE International Conference on.

[23] L. van Hoesel, P. Havinga, A lightweight medium access protocol (lmac) for wireless sensor networks: Reducing preamble transmissions and transceiver state switches.

[24] J. Polastre, J. Hill, D. Culler, Versatile low power media access for wireless sensor networks, in: Proceedings of the 2nd international conference on Embedded networked sensor systems, ACM, 2004, pp. 95-107.

[25] W. Ye, J. Heidemann, D. Estrin, Medium access control with coordinated adaptive sleeping for wireless sensor networks, Networking, IEEE/ACM Transactions on 12 (3) (2004) 493-506.

[26] G. Lu, B. Krishnamachari, C. Raghavendra, An adaptive energy-efficient and low-latency mac for data gathering in wireless sensor networks, in: Parallel and Distributed Processing Symposium, 2004. Proceedings. 18th International, Ieee, 2004, p. 224.

[27] I. Rhee, A. Warrier, M. Aia, J. Min, M. Sichitiu, Z-mac: a hybrid mac for wireless sensor networks, IEEE/ACM Transactions on Networking (TON) 16 (3) (2008) 511-524.

[28] Q. Han, S. Mehrotra, N. Venkatasubramanian, Energy efficient data collection in distributed sensor environments, in: Distributed Computing Systems, 2004. Proceedings. 24th International Conference on, IEEE, 2004 , pp. 590-597.

[29] J. Paradiso, T. Starner, Energy scavenging for mobile and wireless electronics, Pervasive Computing, IEEE 4 (1) (2005) $18-27$.

[30] S. Sudevalayam, P. Kulkarni, Energy harvesting sensor nodes: Survey and implications, Communications Surveys \& Tutorials, IEEE 13 (3) (2011) 443-461.

[31] Powercast corporation, p2000 series $902 \quad 928 \mathrm{mhz}$ powerharvester development kit. http://www.powercastco.com/products/development-kits/.

[32] A. A. Syed, Y. Cho, J. Heidemann, Energy transference for sensornets, in: Proceedings of the 8th ACM Conference on Embedded Networked Sensor Systems, ACM, 2010, pp. 397-398.

[33] Witricity- http://www.witricity.com/.

[34] J. Hsu, S. Zahedi, A. Kansal, M. Srivastava, V. Raghunathan, Adaptive duty cycling for energy harvesting systems, in: Proceedings of the 2006 international symposium on Low power electronics and design, ACM, 2006, pp. 180-185.

[35] C. M. Vigorito, D. Ganesan, A. G. Barto, Adaptive control of duty cycling in energy-harvesting wireless sensor networks, in: Sensor, Mesh and Ad Hoc Communications and Networks, 2007. SECON'07. 4th Annual IEEE Communications Society Conference on, IEEE, 2007, pp. 21-30.

[36] D. Niyato, E. Hossain, A. Fallahi, Sleep and wakeup strategies in solar-powered wireless sensor/mesh networks: Performance analysis and optimization, Mobile Computing, IEEE Transactions on 6 (2) (2007) 221-236.

[37] S. Goel, T. Imielinski, Prediction-based monitoring in sensor networks: taking lessons from mpeg, ACM SIGCOMM Computer Communication Review 31 (5) (2001) 82-98.

[38] A. Deshpande, C. Guestrin, S. Madden, J. Hellerstein, W. Hong, Model-driven data acquisition in sensor networks, in: Proceedings of the Thirtieth international conference on Very large data bases-Volume 30, VLDB Endowment, 2004 , pp. $588-599$.

[39] S. Basagni, A. Carosi, E. Melachrinoudis, C. Petrioli, Z. Wang, Controlled sink mobility for prolonging wireless sensor networks lifetime, Wireless Networks 14 (6) (2008) 831-858.

[40] F. Ye, H. Luo, J. Cheng, S. Lu, L. Zhang, A two-tier data dissemination model for large-scale wireless sensor networks, in: Proceedings of the 8th annual international conference on Mobile computing and networking, ACM, 2002, pp. 148-159.

[41] W. Zhao, M. Ammar, E. Zegura, A message ferrying approach for data delivery in sparse mobile ad hoc networks, in: Proceedings of the 5th ACM international symposium on Mobile ad hoc networking and computing, ACM, 2004, pp. 
$187-198$

[42] R. Shah, S. Roy, S. Jain, W. Brunette, Data mules: Modeling and analysis of a three-tier architecture for sparse sensor networks, Ad Hoc Networks 1 (2-3) (2003) 215-233.

[43] P. Juang, H. Oki, Y. Wang, M. Martonosi, L. Peh, D. Rubenstein, Energy-efficient computing for wildlife tracking: Design tradeoffs and early experiences with zebranet, in: ACM Sigplan Notices, Vol. 37, ACM, 2002, pp. 96-107.

[44] Uc berkeley smartdust project- http://robotics.eecs.berkeley.edu/ pister/smartdust/.

[45] Memsic wireless modules- http://www.memsic.com/products/wireless-sensor-networks/wireless-modules.html.

[46] Crossbow telosb mote- http://bullseye.xbow.com:81/products/.

[47] J. Sheu, P. Cheng, K. Hsieh, Design and implementation of a smart mobile robot, in: Wireless And Mobile Computing, Networking And Communications, 2005.(WiMob'2005), IEEE International Conference on, Vol. 3, IEEE, 2005, pp. $422-429$.

[48] A. LaMarca, W. Brunette, D. Koizumi, M. Lease, S. Sigurdsson, K. Sikorski, D. Fox, G. Borriello, Making sensor networks practical with robots, Pervasive Computing (2002) 615-622.

[49] J. Polastre, R. Szewczyk, D. Culler, Telos: enabling ultra-low power wireless research, in: Information Processing in Sensor Networks, 2005. IPSN 2005. Fourth International Symposium on, Ieee, 2005, pp. 364-369.

[50] R. King, D. Law, K. Edmondson, C. Fetzer, G. Kinsey, H. Yoon, R. Sherif, N. Karam, 40\% efficient metamorphic gainp/gainas/ge multijunction solar cells, Applied physics letters 90 (18) (2007) 183516-183516.

[51] World radiation data centre - http://wrdc.mgo.rssi.ru/.

[52] K. Lin, J. Yu, J. Hsu, S. Zahedi, D. Lee, J. Friedman, A. Kansal, V. Raghunathan, M. Srivastava, Heliomote: enabling long-lived sensor networks through solar energy harvesting, in: Proceedings of the 3rd international conference on Embedded networked sensor systems, ACM, 2005, pp. 309-309.

[53] C. Alippi, C. Galperti, An adaptive system for optimal solar energy harvesting in wireless sensor network nodes, Circuits and Systems I: Regular Papers, IEEE Transactions on 55 (6) (2008) 1742-1750.

[54] T. Esram, P. L. Chapman, Comparison of photovoltaic array maximum power point tracking techniques, Energy conversion, IEEE transactions on 22 (2) (2007) 439-449.

[55] D. Li, P. Chou, Maximizing efficiency of solar-powered systems by load matching, in: Low Power Electronics and Design, 2004. ISLPED'04. Proceedings of the 2004 International Symposium on, IEEE, 2004, pp. 162-167.

[56] J. Taneja, J. Jeong, D. Culler, Design, modeling, and capacity planning for micro-solar power sensor networks, in: Proceedings of the 7th international conference on Information processing in sensor networks, IEEE Computer Society, 2008, pp. 407-418.

[57] T. Wark, P. Corke, P. Sikka, L. Klingbeil, Y. Guo, C. Crossman, P. Valencia, D. Swain, G. Bishop-Hurley, Transforming agriculture through pervasive wireless sensor networks, Pervasive Computing, IEEE 6 (2) (2007) 50-57.

[58] F. I. Simjee, P. H. Chou, Efficient charging of supercapacitors for extended lifetime of wireless sensor nodes, Power Electronics, IEEE Transactions on 23 (3) (2008) 1526-1536.

[59] C. Park, P. Chou, Ambimax: Autonomous energy harvesting platform for multi-supply wireless sensor nodes, in: Sensor and Ad Hoc Communications and Networks, 2006. SECON'06. 2006 3rd Annual IEEE Communications Society on, Vol. 1, IEEE, 2006, pp. 168-177.

[60] F. Simjee, P. Chou, Everlast: long-life, supercapacitor-operated wireless sensor node, in: Low Power Electronics and Design, 2006. ISLPED'06. Proceedings of the 2006 International Symposium on, IEEE, 2006, pp. 197-202.

[61] M. Minami, T. Morito, H. Morikawa, T. Aoyama, Solar biscuit: A battery-less wireless sensor network system for environmental monitoring applications, in: The 2nd International Workshop on Networked Sensing Systems, 2005.

[62] P. Stanley-Marbell, D. Marculescu, Sunflower: Full-system, embedded microarchitecture evaluation, in: High Performance Embedded Architectures and Compilers, Springer, 2007, pp. 168-182.

[63] Turtlenet - http://prisms.cs.umass.edu/dome/turtlenet.

[64] P. Dutta, J. Hui, J. Jeong, S. Kim, C. Sharp, J. Taneja, G. Tolle, K. Whitehouse, D. Culler, Trio: enabling sustainable and scalable outdoor wireless sensor network deployments, in: Proceedings of the 5th international conference on Information processing in sensor networks, ACM, 2006, pp. 407-415.

[65] D. Dondi, A. Di Pompeo, C. Tenti, T. Rosing, Shimmer: A wireless harvesting embedded system for active ultrasonic structural health monitoring, in: Sensors, 2010 IEEE, IEEE, 2010, pp. 2325-2328.

[66] T. He, S. Krishnamurthy, L. Luo, T. Yan, L. Gu, R. Stoleru, G. Zhou, Q. Cao, P. Vicaire, J. A. Stankovic, et al., Vigilnet: An integrated sensor network system for energy-efficient surveillance, ACM Transactions on Sensor Networks (TOSN) 2 (1) (2006) 1-38.

[67] J. A. Stankovic, T. He, Energy management in sensor networks, Philosophical Transactions of the Royal Society A: Mathematical, Physical and Engineering Sciences 370.

[68] F. Iannello, O. Simeone, U. Spagnolini, Medium access control protocols for wireless sensor networks with energy harvesting, Communications, IEEE Transactions on 60 (5) (2012) 1381-1389.

[69] C. E. Perkins, E. M. Royer, Ad-hoc on-demand distance vector routing, in: Mobile Computing Systems and Applications, 1999. Proceedings. WMCSA'99. Second IEEE Workshop on, IEEE, 1999, pp. 90-100.

[70] Y. Peng, Z. Li, W. Zhang, D. Qiao, Prolonging sensor network lifetime through wireless charging, in: Real-Time Systems Symposium (RTSS), 2010 IEEE 31st, IEEE, 2010, pp. 129-139.

[71] S. Guo, C. Wang, Y. Yang, Mobile data gathering with wireless energy replenishment in rechargeable sensor networks, in: INFOCOM, 2013 Proceedings IEEE, IEEE, 2013, pp. 1932-1940.

[72] Z. Li, Y. Peng, W. Zhang, D. Qiao, J-roc: A joint routing and charging scheme to prolong sensor network lifetime, in: Network Protocols (ICNP), 2011 19th IEEE International Conference on, IEEE, 2011, pp. 373-382.

[73] K. Li, H. Luan, C.-C. Shen, Qi-ferry: Energy-constrained wireless charging in wireless sensor networks, in: Wireless 
Communications and Networking Conference (WCNC), 2012 IEEE, IEEE, 2012, pp. 2515-2520.

[74] M. Watfa, H. AlHassanieh, S. Selman, Multi-hop wireless energy transfer in wsns, Communications Letters, IEEE (99) (2011) $1-3$.

[75] J. Wu, M. Cardei, F. Dai, S. Yang, Extended dominating set and its applications in ad hoc networks using cooperative communication, Parallel and Distributed Systems, IEEE Transactions on 17 (8) (2006) 851-864.

[76] Z. Yuanyuan, X. Jia, H. Yanxiang, Energy efficient distributed connected dominating sets construction in wireless sensor networks, in: Proceedings of the 2006 international conference on Wireless communications and mobile computing, ACM, 2006, pp. 797-802.

[77] P. M. Wightman, M. A. Labrador, A3: A topology construction algorithm for wireless sensor networks, in: Global Telecommunications Conference, 2008. IEEE GLOBECOM 2008. IEEE, IEEE, 2008, pp. 1-6.

[78] S. Rizvi, H. K. Qureshi, S. Ali Khayam, V. Rakocevic, M. Rajarajan, A1: An energy efficient topology control algorithm for connected area coverage in wireless sensor networks, Journal of Network and Computer Applications 35 (2) (2012) $597-605$.

[79] H. K. Qureshi, S. Rizvi, M. Saleem, S. A. Khayam, V. Rakocevic, M. Rajarajan, Poly: A reliable and energy efficient topology control protocol for wireless sensor networks, Computer Communications 34 (10) (2011) 1235-1242.

[80] P. M. Wightman, M. A. Labrador, Atarraya: A simulation tool to teach and research topology control algorithms for wireless sensor networks, in: Proceedings of the 2nd International Conference on Simulation Tools and Techniques, ICST (Institute for Computer Sciences, Social-Informatics and Telecommunications Engineering), 2009 , p. 26.

[81] T. Yang, C. Kang, G. Nan, An energy-efficient and fault-tolerant convergecast protocol in wireless sensor networks, International Journal of Distributed Sensor Networks 2012.

[82] C. Intanagonwiwat, R. Govindan, D. Estrin, Directed diffusion: a scalable and robust communication paradigm for sensor networks, in: Proceedings of the 6th annual international conference on Mobile computing and networking, ACM, 2000, pp. $56-67$.

[83] J. Choe, K. Kim, Eadd: Energy aware directed diffusion for wireless sensor networks, in: Parallel and Distributed Processing with Applications, 2008. ISPA'08. International Symposium on, IEEE, 2008, pp. 779-783.

[84] Z. Che-Aron, W. Al-Khateeb, F. Anwar, Enfat-aodv: The fault-tolerant routing protocol for high failure rate wireless sensor networks, in: Future Computer and Communication (ICFCC), 2010 2nd International Conference on, Vol. 1, IEEE, 2010, pp. V1-467.

[85] T. He, J. A. Stankovic, C. Lu, T. Abdelzaher, Speed: A stateless protocol for real-time communication in sensor networks, in: Distributed Computing Systems, 2003. Proceedings. 23rd International Conference on, IEEE, 2003, pp. 46-55.

[86] K.-Y. Shin, J. Song, J. Kim, M. Yu, P. S. Mah, Rear: reliable energy aware routing protocol for wireless sensor networks, in: Advanced Communication Technology, The 9th International Conference on, Vol. 1, IEEE, 2007, pp. 525-530.

[87] T. Yang, Y. Sun, J. Taheri, A. Y. Zomaya, Dls: a dynamic local stitching mechanism to rectify transmitting path fragments in wireless sensor networks, Journal of Network and Computer Applications 36 (1) (2013) $306-315$.

[88] C. Schurgers, V. Tsiatsis, M. B. Srivastava, Stem: Topology management for energy efficient sensor networks, in: Aerospace Conference Proceedings, 2002. IEEE, Vol. 3, IEEE, 2002, pp. 3-1099.

[89] X. Yang, N. H. Vaidya, A wakeup scheme for sensor networks: achieving balance between energy saving and end-to-end delay, in: Real-Time and Embedded Technology and Applications Symposium, 2004. Proceedings. RTAS 2004. 10th IEEE, IEEE, 2004, pp. 19-26.

[90] L. Gu, J. A. Stankovic, Radio-triggered wake-up for wireless sensor networks, Real-Time Systems 29 (2-3) (2005) $157-182$.

[91] M. Salajegheh, H. Soroush, A. Kalis, Hymac: Hybrid tdma/fdma medium access control protocol for wireless sensor networks, in: Personal, Indoor and Mobile Radio Communications, 2007. PIMRC 2007. IEEE 18th International Symposium on, IEEE, 2007, pp. 1-5.

[92] H. Yoo, M. Shim, D. Kim, Dynamic duty-cycle scheduling schemes for energy-harvesting wireless sensor networks, Communications Letters, IEEE 16 (2) (2012) 202-204.

[93] A. Sinha, A. Chandrakasan, Dynamic power management in wireless sensor networks, Design \& Test of Computers, IEEE 18 (2) (2001) 62-74.

[94] P. Pillai, K. G. Shin, Real-time dynamic voltage scaling for low-power embedded operating systems, in: ACM SIGOPS Operating Systems Review, Vol. 35, ACM, 2001, pp. 89-102.

[95] S. Liu, Q. Wu, Q. Qiu, An adaptive scheduling and voltage/frequency selection algorithm for real-time energy harvesting systems, in: Design Automation Conference, 2009. DAC'09. 46th ACM/IEEE, IEEE, 2009, pp. 782-787.

[96] S. Herbert, D. Marculescu, Analysis of dynamic voltage/frequency scaling in chip-multiprocessors, in: Low Power Electronics and Design (ISLPED), 2007 ACM/IEEE International Symposium on, IEEE, 2007, pp. 38-43.

[97] D. Chu, A. Deshpande, J. M. Hellerstein, W. Hong, Approximate data collection in sensor networks using probabilistic models, in: Data Engineering, 2006. ICDE'06. Proceedings of the 22nd International Conference on, IEEE, 2006, pp. 48-48.

[98] D. Tulone, S. Madden, Paq: Time series forecasting for approximate query answering in sensor networks, in: Wireless Sensor Networks, Springer, 2006, pp. 21-37.

[99] D. Tulone, S. Madden, An energy-efficient querying framework in sensor networks for detecting node similarities, in: Proceedings of the 9th ACM international symposium on Modeling analysis and simulation of wireless and mobile systems, ACM, 2006, pp. 191-300.

[100] B. Gedik, L. Liu, P. S. Yu, Asap: an adaptive sampling approach to data collection in sensor networks, Parallel and Distributed Systems, IEEE Transactions on 18 (12) (2007) 1766-1783.

[101] P. Padhy, R. K. Dash, K. Martinez, N. R. Jennings, A utility-based sensing and communication model for a glacial sensor network, in: Proceedings of the fifth international joint conference on Autonomous agents and multiagent systems, ACM, 
2006, pp. 1353-1360.

[102] H. S. Kim, T. F. Abdelzaher, W. H. Kwon, Minimum-energy asynchronous dissemination to mobile sinks in wireless sensor networks, in: Proceedings of the 1st international conference on Embedded networked sensor systems, ACM, 2003, pp. 193-204.

[103] J. R. Piorno, C. Bergonzini, D. Atienza, T. S. Rosing, Prediction and management in energy harvested wireless sensor nodes, in: Wireless Communication, Vehicular Technology, Information Theory and Aerospace \& Electronic Systems Technology, 2009. Wireless VITAE 2009. 1st International Conference on, IEEE, 2009, pp. 6-10.

[104] D. K. Noh, L. Wang, Y. Yang, H. K. Le, T. Abdelzaher, Minimum variance energy allocation for a solar-powered sensor system, in: Distributed Computing in Sensor Systems, Springer, 2009, pp. 44-57.

[105] L. Wang, Y. Yang, D. K. Noh, H. K. Le, J. Liu, T. F. Abdelzaher, M. Ward, Adaptsens: an adaptive data collection and storage service for solar-powered sensor networks, in: Real-Time Systems Symposium, 2009, RTSS 2009. 30th IEEE, IEEE, 2009, pp. 303-312.

[106] C. Moser, L. Thiele, D. Brunelli, L. Benini, Adaptive power management for environmentally powered systems, Computers, IEEE Transactions on 59 (4) (2010) 478-491.

[107] W. B. Heinzelman, A. P. Chandrakasan, H. Balakrishnan, An application-specific protocol architecture for wireless microsensor networks, Wireless Communications, IEEE Transactions on 1 (4) (2002) 660-670.

[108] S. P. Ardakani, J. Padget, M. De Vos, Hrts: A hierarchical reactive time synchronization protocol for wireless sensor networks, in: Ad Hoc Networks, Springer, 2014, pp. 47-62.

[109] A. Perrig, R. Szewczyk, J. Tygar, V. Wen, D. E. Culler, Spins: Security protocols for sensor networks, Wireless networks 8 (5) (2002) 521-534.

[110] S. Zhu, S. Setia, S. Jajodia, Leap: Efficient security mechanisms for large-scale distributed sensor networks, ACM Transactions on Sensor Networks (TOSN) 2 (4) (2006) 500-528.

[111] C.-C. Shen, C. Srisathapornphat, C. Jaikaeo, Sensor information networking architecture and applications, Personal communications, IEEE 8 (4) (2001) 52-59.

[112] S. Madden, M. J. Franklin, J. M. Hellerstein, W. Hong, Tag: A tiny aggregation service for ad-hoc sensor networks, SIGOPS Oper. Syst. Rev. 36 (SI) (2002) 131-146. doi:10.1145/844128.844142. URL http://doi.acm.org/10.1145/844128.844142

[113] S. R. Madden, M. J. Franklin, J. M. Hellerstein, W. Hong, Tinydb: An acquisitional query processing system for sensor networks, ACM Transactions on Database Systems (TODS) 30 (1) (2005) 122-173. 